\title{
Polynomial Invariants of the Euclidean Group Action on Multiple Screws
}

by

\section{Deborah Crook}

A thesis submitted to the Victoria University of Wellington in fulfilment of the requirements for the degree of Master of Science in Mathematics

Victoria University of Wellington

August 2009 


\begin{abstract}
In this work, we examine the polynomial invariants of the special Euclidean group in three dimensions, $S E(3)$, in its action on multiple screw systems. We look at the problem of finding generating sets for these invariant subalgebras, and also briefly describe the invariants for the standard actions on $\mathbb{R}^{n}$ of both $S E(3)$ and $S O(3)$. The problem of the screw system action is then approached using SAGBI basis techniques, which are used to find invariants for the translational subaction of $S E(3)$, including a full basis in the one and two-screw cases. These are then compared to the known invariants of the rotational subaction. In the one and two-screw cases, we successfully derive a full basis for the $S E(3)$ invariants, while in the three-screw case, we suggest some possible lines of approach.
\end{abstract}




\section{Contents}

1 Introduction 1

2 Introduction to $S E(3)+\mathbf{5}$

2.1 The special Euclidean group . . . . . . . . . . . 5

2.2 Representation of joints . . . . . . . . . . . 7

2.3 Lie algebras . . . . . . . . . . . . . . . . 8

3 Gröbner Bases $\quad 13$

3.1 Monomial orderings . . . . . . . . . . . . . 13

3.2 Gröbner bases . . . . . . . . . . . . . . . 16

3.2.1 Buchberger's algorithm . . . . . . . . . . . 18

4 Finite Group Actions 20

4.1 Invariant polynomials . . . . . . . . . . . . . 20 
4.2 Syzygies . . . . . . . . . . . . . . . . . 21

4.3 Finding generators $\ldots \ldots \ldots \ldots \ldots$

4.4 Hironaka decompositions . . . . . . . . . . . . . . 27

4.5 Reductive groups . . . . . . . . . . . . . . . . . 29

5 SAGBI Bases 32

5.1 Introduction . . . . . . . . . . . . . . . 32

5.2 Constructing a SAGBI basis . . . . . . . . . . . 35

5.2.1 SAGBI basis construction algorithm . . . . . . 36

$\begin{array}{lll}6 & S O(n) \text { invariants } & 38\end{array}$

6.1 The action on two vectors . . . . . . . . . . . . . 39

6.2 The action on $m$ vectors $\ldots \ldots \ldots$. . . . . . . . . 42

6.3 Capelli's identity . . . . . . . . . . . . . . . . . 43

6.4 A SAGBI basis . . . . . . . . . . . . . . . 50

6.5 The standard action of $S E(n) \ldots \ldots \ldots \ldots$

6.6 Syzygies of $S O(n) \ldots \ldots \ldots \ldots \ldots \ldots$ 
7.1 A basis for invariants . . . . . . . . . . . . . 62

7.2 Application to $S O(n) \ldots \ldots \ldots \ldots \ldots$

7.3 Single-screw case $\ldots \ldots \ldots \ldots \ldots$

7.4 Double and triple screw cases $\ldots \ldots \ldots \ldots$ 


\section{Chapter 1}

\section{Introduction}

The Special Euclidean group $S E(3)$ is the set of all three-dimensional translations and rotations; that is, rigid body transformations in three-dimensional space. These transformations can be used to describe motions in many different problems in physics and engineering. For example, these motions may be made by a mechanism such as a robotic arm, something which is found in many different industries. We assume that a simple arm can be modelled as a series of joints, each capable of rotation, translation, or a combination of the two, linked together by rigid elements. Usually there is some sort of manipulator at the tip, called the end-effector. In particular, these motions depend on several variables.

The infinitesimal motion of these mechanisms can be represented by screw systems, which are k-dimensional subspaces of the Lie algebra $\mathfrak{s e}(3)$. We are interested in classifying different screw systems, corresponding to different robotic arms, by looking at their behaviour under the adjoint action. This 
is the action of $S E(3)$ on its Lie algebra $\mathfrak{s e}(3)$ that, geometrically speaking, corresponds to a change of coordinates in the ambient space. One way to distinguish various orbits of this action is to look at its polynomial invariants (though this does not always work; for example, in a reductive group, invariants will only distinguish between closed orbits), for which it is useful to know a generating set for all possible invariants. Hence the aim of this paper is to try to find generating sets for invariants of the adjoint action of $S E(3)$ on multi-degree screw systems, corresponding to robotic arms with multiple joints. Unfortunately, $S E(3)$ is not a reductive group, as the adjoint representation is not completely reducible, and so most of the standard invariant theory for finite and infinite reductive groups does not directly apply, although we do know that the invariant ring of the adjoint action is finitely generated [4].

The approach in this paper is to divide the action of $S E(3)$ into translational and rotational subactions, and attempt to find the invariants for each. This approach rests on a result due to Vasconcelos [14], who gives a method for finding generating sets for invariant subrings of many groups, including non-reductive ones, although it does not work when applied directly to the action of $S E(3)$. I used this method to find the translational invariants; the results for the rotational invariants come from the standard action of $S O(3)$, and may be found in [15]. The next step is to use the invariants of both subactions in order to find the set of special Euclidean invariants, which is the intersection of the two previous invariant rings. This is successful in the 
case of one- and two-screw systems, where I have found a full set of generating invariants for $S E(3)$, but not for the three-screw system, where only the rotational and some of the translational invariants are found. Some explorations of possible ways to find the full set of special Euclidean invariants are also included. The calculations use the notion of SAGBI bases for subalgebras, which are more fully explained in Section 3, but have a number of useful properties in analogue with Gröbner bases for ideals.

The second chapter deals with $S E(3)$ and $\mathfrak{s e}(3)$, and presents a more complete setup of the problem. It also explains the connection between screw systems and mechanical joints, and how the adjoint action affects them. The third chapter contains a brief review of relevant algebraic notions; namely, monomial orderings and Gröbner bases, including a construction algorithm. The next chapter continues with a review of some classical invariant theory for finite and reductive groups, including Hilbert's finiteness theorem and construction methods for finding a generating set for the invariant subring in the finite case. This is presented by way of comparison and a general overview on the subject; it is not directly used to find the $S O(3)$ or $S E(3)$ invariants.

Chapter 5 introduces the notion of SAGBI bases, due to Robbiano and Sweedler [10], and explains some of their properties, as well as presenting a construction algorithm similar to the Gröbner basis one in Chapter 2 . Then Chapter 6 deals with the invariant ring of $S O(n)$, which is needed for finding the invariants of the rotational subaction of $S E(3)$. It goes through 
the classical derivation of a generating set for the $S O(n)$ invariants, due to Weyl [15], then presents a SAGBI basis for the same invariant ring. This is then used to find a SAGBI basis for the standard action of $S E(3)$ on $\mathbb{R}^{n}$. Finally, a Gröbner basis is given for the set of all algebraic relations amongst the $S O(3)$ invariants.

The last chapter presents the methods used to find the translational invariants, and the generating sets I have calculated for the translational invariants in the one and two-screw cases, as well as some invariants for the three-screw case. The first two cases also have a derivation of the set of full special Euclidean invariants, which fails for the last case, although some invariants can still be found by inspection. Finally, there is a discussion of possible methods for finding the full invariants in the three-screw case, although these were not fully explored. 


\section{Chapter 2}

\section{Introduction to $S E(3)$}

\subsection{The special Euclidean group}

The special orthogonal group $S O(3)$ is the group of rotations about the origin in three dimensions. Since rotations preserve both lengths and angles, the elements of $S O(3)$ preserve the positive definite bilinear form $\mathbf{x}^{T} \mathbf{x}=\mathbf{x} \cdot \mathbf{x}$ in three-dimensional Euclidean space. This means that they can be written as $3 \times 3$ orthogonal matrices. Furthemore, they must also preserve orientation, so the matrices must have determinant 1.

The special Euclidean group, $S E(3)$, is the semi-direct product of $S O(3)$ and the group of translations in three dimensions, $\mathbb{R}^{3}$. This is sometimes called the set of rigid-body transformations, as it consists of all possible transformations that can be made by an actual physical rigid object in threedimensional space, which can rotate and translate, but cannot change either its size or its orientation. 
This product is a 6-dimensional Lie group, which is defined as a smooth manifold $G$ with a group structure, such that the group operations:

$$
\begin{array}{r}
.: G \times G \longrightarrow G \\
-1: \quad G \longrightarrow G
\end{array}
$$

are continuous differentiable mappings with respect to the manifold structure.

In the case of $S E(3)$, the group product is given by:

$$
\left(R_{1}, \mathbf{r}_{1}\right) \cdot\left(R_{2}, \mathbf{r}_{2}\right)=\left(R_{1} R_{2}, \mathbf{r}_{1}+R \mathbf{r}_{2}\right)
$$

and it has an affine action on $\mathbb{R}^{3}$ given by:

$$
(R, \mathbf{r}) \mathbf{x}=R \mathbf{x}+\mathbf{r}
$$

This can also be expressed in terms of a linear matrix representation:

$$
\left(\begin{array}{ll}
R & \mathbf{r} \\
0 & 1
\end{array}\right)\left(\begin{array}{c}
\mathbf{x} \\
1
\end{array}\right)=\left(\begin{array}{c}
R \mathbf{x}+\mathbf{r} \\
1
\end{array}\right)
$$

acting on the subset of $\mathbb{R}^{4}$ with last coordinate 1 , isomorphic to $\mathbb{R}^{3}$.

Then, for a moving rigid body (such as the end-effector of a robotic arm), we can specify two different coordinate frames: a home, or base, frame of reference that is fixed in the ambient space around the mechanism; and a coordinate system embedded in the body itself. If a point has coordinates $\mathbf{p}$ in the reference frame, and coordinates $\mathbf{p}^{\prime}$ in the body frame, then these are linked by $\mathbf{p}=A \mathbf{p}^{\prime}$ for some $A \in S E(3)$. Now we change the ambient 
coordinates by a transformation $T \in S E(3)$, so that a point $\mathbf{p}$ in the old frame of reference has coordinates $\mathbf{p}_{1}=T^{-1} \mathbf{p}$ in the new one.

So if $A$ is the transformation taking $\mathbf{p}^{\prime}$ to $\mathbf{p}$, then in the new frame of reference:

$$
\begin{aligned}
A \mathbf{p}^{\prime} & =\mathbf{p} \\
A\left(T \mathbf{p}_{1}^{\prime}\right) & =T \mathbf{p}_{1} \\
\left(T^{-1} A T\right) \mathbf{p}_{1}^{\prime} & =\mathbf{p}_{1}
\end{aligned}
$$

So the change of coordinates takes $A \mapsto T^{-1} A T$, which is the conjugation mapping in $S E(3)$.

\section{$2.2 \quad$ Representation of joints}

Chasles's Theorem states that every element of $S E(3)$ is equivalent to some finite screw form; that is, a rotation about an axis combined with a translation along it. If the axis passes through the origin, then the screw form can be written as:

$$
\left(R(\theta, \hat{\mathbf{v}}), \frac{\theta}{2 \pi} p \hat{\mathbf{v}}\right)
$$

where $\hat{\mathbf{v}}$ is a unit vector parallel to the axis, $\theta$ is the angle of rotation, and $p$ is the pitch of the screw, defined as the distance travelled along the axis

for one complete revolution around it. If the axis does not pass through the 
origin, take $\mathbf{u}$ to be the position vector of an arbitrary point on the axis. Then the screw form can be written as:

$$
\left(I_{3}, \mathbf{u}\right)\left(R(\theta, \hat{\mathbf{v}}), \frac{\theta}{2 \pi} p \hat{\mathbf{v}}\right)\left(I_{3},-\mathbf{u}\right)=\left(R, \frac{\theta}{2 \pi} p \hat{\mathbf{v}}+\left(I_{3}-R\right) \mathbf{u}\right)
$$

by translating the axis to the origin, finding the screw form, and translating it back.

A common form for a mechanical joint is a pair of identically-shaped surfaces, placed in contact with each other at all points, and free to move relative to each other while maintaining that contact. Some examples are a pair of nested spheres, which can form a ball-and-socket joint; two contacting planes; nested surfaces of revolution or translation; or nested helicoidal surfaces. These are called Reuleaux lower pairs [8]. They consist of surfaces in $\mathbb{R}^{3}$ that are invariant under a Lie subgroup of $S E(3)$, with the dimension of the subgroup being the same as the number of degrees of freedom of the joint. Of particular interest are those with only one degree of freedom: revolute, prismatic (translational) and helical joints, formed from surfaces of rotation, translation, and helicoidal surfaces respectively. These all correspond to 1dimensional subgroups of $S E(3)$ generated by a single element of $S E(3)$. Hence all these joints can be represented by screw forms.

\subsection{Lie algebras}

Given a Lie group, we can construct its Lie algebra by taking the space of all possible tangent vectors at the identity to paths in the group passing 
through it (up to a natural equivalence). This space is an algebra with an anticommutative multiplication operator, called the Lie bracket and usually denoted by $[\cdot, \cdot]$. (More on the theory of Lie groups and their algebras can be found in [6]). The Lie algebra of $S O(3)$ is denoted $\mathfrak{s o}(3)$, and consists of all $3 \times 3$ antisymmetric matrices. Geometrically, it is the set of infinitesimal rotations, and its Lie bracket is just the commutator $[X, Y]=X Y-Y X$. Likewise, the Lie algebra of $S E(3)$ is $\mathfrak{s e}(3)$, the product of $\mathfrak{s o}(3)$ and the set of infinitesimal translations, $\mathfrak{t}(3)$, which is isomorphic to $\mathbb{R}^{3}$. Every element of $\mathfrak{s e}(3)$, called a twist, is associated with a left-invariant vector field on $S E(3)$, generated by using left-multiplication to map the tangent space at the identity to a tangent space at any other point of $S E(3)$. Hence for the infinitesimal motion of a rigid body, there is an element of $\mathfrak{s e}(3)$ that generates the vector field that contains that motion, which can be found by mapping it back to the identity.

Now we consider a way of writing the elements of $\mathfrak{s e}(3)$. The derivation here follows that in [11]. Let $\mathbf{p}=(x, y, z)$ be a point on a rigid body that is moving along a path in space, so that its position at time $t$ is $\mathbf{p}(t)=(R(t), \mathbf{r}(t)) \mathbf{p}(0)$, with $(R(0), \mathbf{r}(0))$ being the identity in $S E(3)$.

Let $\mathbf{s}=(\dot{R}(0), \dot{\mathbf{r}}(0))=(\Omega, \mathbf{v})$ be the Lie algebra element corresponding to this motion in $\mathfrak{s e}(3)$. Then the instantaneous velocity of $\mathbf{p}$ at time 0 is:

$$
\begin{aligned}
\dot{\mathbf{p}}(0) & =(\dot{x}(0), \dot{y}(0), \dot{z}(0))^{T} \\
& =\dot{R}(0)(x(0), y(0), z(0))+\dot{\mathbf{r}}(0) \\
& =\Omega \mathbf{p}(0)+\mathbf{v}
\end{aligned}
$$


Since $\Omega$ is a $3 \times 3$ antisymmetric matrix, it can be written as:

$$
\Omega=\left(\begin{array}{ccc}
0 & -\omega_{z} & \omega_{y} \\
\omega_{z} & 0 & -\omega_{x} \\
-\omega_{y} & \omega_{x} & 0
\end{array}\right)
$$

for some $\boldsymbol{\omega} \in \mathbb{R}$, giving $\Omega \mathbf{p}=\boldsymbol{\omega} \times \mathbf{p}$.

So $(2.7)$ can be written as:

$$
\dot{\mathbf{p}}=\omega \times \mathbf{p}+\mathbf{v}
$$

The $\boldsymbol{\omega} \times \mathbf{p}$ part of this is purely rotational motion, as $\Omega \in \mathfrak{s o}(3)$, and the last part is purely translational motion in the direction of $\mathbf{v}$.

The 6-dimensional coordinates $(\boldsymbol{\omega}, \mathbf{v})$, called Plücker coordinates, give one way of writing the elements of $\mathfrak{s e}(3)$. For a zero-pitch twist, $\boldsymbol{\omega}$ is parallel to the axis of rotation, and $\mathbf{v}$ is the normal to the plane containing both $\mathbf{u}$ and $\boldsymbol{\omega}$, i.e. the plane containing the origin and the axis of rotation. For a screw with non-zero pitch, $\mathbf{v}$ has a component parallel to the axis of rotation as well.

As with any Lie group, $S E(3)$ has a group action on its Lie algebra by means of the adjoint representation, $S E(3): \mathfrak{s e}(3) \rightarrow \mathfrak{s e}(3), \mathbf{s} \mapsto A \mathbf{s} A^{-1}$, where $A \in S E(3)$. Using the Plücker coordinates, this can be written as:

$$
\left(\begin{array}{cc}
R & 0 \\
T R & R
\end{array}\right)\left(\begin{array}{l}
\omega \\
\mathbf{v}
\end{array}\right)=\left(\begin{array}{c}
R \omega \\
T R \omega+R \mathbf{v}
\end{array}\right)
$$

where $R \in S O(3)$, and

$$
T=\left(\begin{array}{ccc}
0 & -t_{z} & t_{y} \\
t_{z} & 0 & -t_{x} \\
-t_{y} & t_{x} & 0
\end{array}\right)
$$


represents a translation $\mathbf{t}=\left(t_{x}, t_{y}, t_{z}\right) \in \mathbb{R}^{3}$. This contains subactions of just the translations (taking $R=I_{3}$ ):

$$
\left(\begin{array}{cc}
I & 0 \\
T R & I
\end{array}\right)\left(\begin{array}{l}
\omega \\
\mathbf{v}
\end{array}\right)=\left(\begin{array}{c}
\omega \\
T \omega+\mathbf{v}
\end{array}\right)
$$

and just the rotations (taking $T=0$ ):

$$
\left(\begin{array}{ll}
R & 0 \\
0 & R
\end{array}\right)\left(\begin{array}{l}
\omega \\
\mathbf{v}
\end{array}\right)=\left(\begin{array}{l}
R \omega \\
R \mathbf{v}
\end{array}\right)
$$

The latter is isomorphic to a double action of $S O(3)$ on its Lie algebra $\mathfrak{s o}(3)$.

As mentioned before, an element of $\mathfrak{s e}(3)$ is associated with a left-invariant vector field. Taking the path along this vector field that passes through the identity then gives a 1-parameter subgroup of $S E(3)$ associated with that twist. The mapping from twist to subgroup is called the exponential mapping. This means that a mechanical joint with a one degree of freedom can be described in terms of a twist that generates its associated subgroup.

Given a twist, the subspace in $\mathfrak{s e}(3)$ that it generates can be represented by a point in the projective space of $\mathfrak{s e}(3)$, called a screw. Any multiple of the twist will give the same point in projective space, and furthermore, it will generate the same 1-parameter subgroup in $S E(3)$, by tracing out the same path at a different rate, under the exponential mapping. This means that it is more sensible to associate the 1-parameter subgroup, and the joint it represents, with the screw rather than the twist. This is called a screw system. A mechanism with multiple joints is described by a screw system of 
degree $k$, which is a $(k-1)$-dimensional subspace of the projective space of $\mathfrak{s e}(3)$, corresponding to a $k$-dimensional subspace in $\mathfrak{s e}(3)$, spanned by the twists of each joint. This screw system describes the infinitesimal motion of the end-effector of the mechanism.

We can consider two screw systems to be the same if there is a change of coordinates taking one into the other; i.e., the adjoint action of $S E(3)$ takes the set of twists for one screw system to a set that generates the second. In fact, the choice of twists to represent a screw system is not unique, so there are many more actions, such as a change of basis, that will give the same screw system, but the focus of this paper is the adjoint action.

So we want to be able to distinguish between different orbits of $S E(3)$ in its Lie algebra in order to help classify different screw systems. One way to do this is by looking at the polynomial invariants in the coordinates of $\mathfrak{s e}(3)$.

For example, in the single screw case, the different orbits are distinguished by looking at the pitch, which is equal to $\frac{\boldsymbol{\omega} \cdot \mathbf{v}}{\boldsymbol{\omega} \cdot \boldsymbol{\omega}}$. This is the ratio of two polynomial invariants, and it separates all orbits, except those for where $\boldsymbol{\omega} \cdot \boldsymbol{\omega}=0$. On this subspace, however, $\mathbf{v} \cdot \mathbf{v}$ becomes an invariant, and can be used to separate the remaining orbits. 


\section{Chapter 3}

\section{Gröbner Bases}

A single-variable polynomial ring has some advantages over the multi-variate case when it comes to finding generating sets for ideals. Any ideal in $k[x]$ is generated by a single polynomial, and dividing by that polynomial gives a unique remainder, a property that can be used to test other polynomials for membership in that ideal. However, these properties are in general not true for ideals in multi-variable polynomial rings, though some of them can be recovered by using particular bases, known as Gröbner bases. In order to define these, though, we first need to have a standard way of writing each polynomial; that is, an unequivocal order for its terms.

\subsection{Monomial orderings}

A monomial in multiple variables can be written as $\mathbf{x}^{\alpha}:=x_{1}^{\alpha_{1}} \ldots x_{n}^{\alpha_{n}}$, for some $\alpha \in \mathbb{Z}_{\geq 0}^{n}$, which is the set of non-negative integers. The total degree 
of this monomial is then $|\alpha|:=\alpha_{1}+\ldots+\alpha_{n}$. In the single-variable case, there is an obvious and unique way to order these monomials: namely, by their degrees. With multiple variables it is much less clear, and there may be several suitable ways. However, we can choose a few basic properties that these orderings should have. If $k\left[x_{1}, \ldots, x_{n}\right]$ is the set of all polynomials in $x_{1}, \ldots, x_{n}$ with coefficients drawn from the field $k$, then:

Definition 3.1.1 A monomial ordering on $k\left[x_{1}, \ldots, x_{n}\right]$ is a relation $>$ on the set of monomials $\left\{\boldsymbol{x}^{\alpha}: \alpha \in \mathbb{Z}_{\geq 0}^{n}\right\}$ in $k\left[x_{1}, \ldots, x_{n}\right]$, such that:

1. $>$ is a total ordering.

2. If $\boldsymbol{x}^{\alpha}>\boldsymbol{x}^{\beta}$ and $\gamma \in \mathbb{Z}_{\geq 0}^{n}$, then $\boldsymbol{x}^{\alpha} \boldsymbol{x}^{\gamma}>\boldsymbol{x}^{\beta} \boldsymbol{x}^{\gamma}$.

3. Every non-empty set of monomials in $k\left[x_{1}, \ldots, x_{n}\right]$ has a least element under $>$.

Equivalently, this ordering can be defined on $\mathbb{Z}_{\geq 0}^{n}$, with similar properties. Some examples of common monomial orderings are:

1. Lexicographic Order Let $\alpha=\left(\alpha_{1}, \ldots, \alpha_{n}\right)$ and $\beta=\left(\beta_{1}, \ldots, \beta_{n}\right)$ be in $\mathbb{Z}_{\geq 0}^{n}$, and suppose that $k \in\{1, \ldots, n\}$ is the smallest number such that $\alpha_{k} \neq \beta_{k}$. Then

$$
\alpha>_{l e x} \beta, \quad \text { or } \quad \mathbf{x}^{\alpha}>_{l e x} \mathbf{x}^{\beta}, \quad \text { if } \quad \alpha_{k}-\beta_{k}>0
$$


e.g. in $k\left[x_{1}, x_{2}, x_{3}\right], x_{1}^{6}>x_{1}^{5}>x_{1} x_{2}^{6}>x_{2}^{9}$.

This is based on the ordering $x_{1}>x_{2}>\ldots>x_{n}$. A reordering of the variables will give a different lexicographic ordering on the set of monomials in $k\left[x_{1}, \ldots, x_{n}\right]$.

2. Graded Lexicographic Order Let $\alpha, \beta \in \mathbb{Z}_{\geq 0}^{n}$. Then $\alpha>_{\text {grlex }} \beta$, or $\mathbf{x}^{\alpha}>_{\text {grlex }} \mathbf{x}^{\beta}$, if $|\alpha|>|\beta|$ or $|\alpha|=|\beta|$ and $\alpha>_{\text {lex }} \beta$.

e.g $x_{2}^{6}>x_{1}^{5}>x_{1}^{4} x_{2}$.

3. Graded Reverse Lexicographic Order Let $\alpha, \beta \in \mathbb{Z}_{\geq 0}^{n}$, and let $k \in\{1, \ldots, n\}$ be the greatest number such that $\alpha_{k} \neq \beta_{k}$. Then $\alpha>_{\text {grevlex }} \beta$, or $\mathbf{x}^{\alpha}>_{\text {grevlex }} \mathbf{x}^{\beta}$

$$
\text { if } \quad|\alpha|>|\beta| \quad \text { or } \quad|\alpha|=|\beta| \quad \text { and } \quad \alpha_{k}-\beta_{k}<0
$$

e.g. $x_{2}^{6}>x_{1}^{5}>x_{1}^{4} x_{3}>x_{1}^{3} x_{3}^{2}>x_{2}^{3} x_{3}^{2}$.

Again, this is based on the ordering $x_{1}>\ldots>x_{n}$.

A monomial ordering is called an elimination ordering if the variables can be divided into two sets: $\left\{x_{1}, \ldots, x_{n}\right\} \cup\left\{y_{1}, \ldots, y_{m}\right\}$, for which any monomial containing any one of $x_{1}, \ldots, x_{n}$ is greater than all monomials in $k\left[y_{1}, \ldots, y_{m}\right]$. Lexicographic ordering is an example of this, as is a product order, where two separate orderings are used on $k\left[x_{1}, \ldots, x_{n}\right]$ and $k\left[y_{1}, \ldots, y_{m}\right]$. In this 
case, monomials in $k\left[x_{1}, \ldots, x_{n}, y_{1}, \ldots, y_{m}\right]$ are sorted by their ordering with respect to $x_{1}, \ldots, x_{n}$, with ties being broken by the ordering with respect to $y_{1}, \ldots, y_{m}$.

Once a monomial ordering is chosen, we can sort all the terms of a given polynomial $f$, and find the leading term $L T(f)$ (which includes the coefficient). Then the leading term of $f$ without its coefficient is the leading monomial, $L M(f)$, and, taking this to be $\mathbf{x}^{\alpha}$ for some $\alpha \in \mathbb{Z}_{\geq 0}^{n}$, we define the multidegree of $f$ to be $\alpha$, denoted $\operatorname{deg} f$. Finally, for a subset $I \subset k\left[x_{1}, \ldots, x_{n}\right]$, $L T(I):=\{L T(f): f \in I\}$ is the set of all its leading terms, and the ideal they generate is denoted $\langle L T(I)\rangle$.

\subsection{Gröbner bases}

The concept of a Gröbner basis can now be defined:

Definition 3.2.1 Let $I \subset k\left[x_{1}, \ldots, x_{n}\right]$ be an ideal, and choose some monomial ordering. A finite subset $G=\left\{g_{1}, \ldots, g_{s}\right\}$ of $I$ is a Gröbner basis if

$$
\left\langle L T\left(g_{1}\right), \ldots, L T\left(g_{s}\right)\right\rangle=\langle L T(I)\rangle
$$

This means that the leading term of any element of $I$ is divisible by some $L T\left(g_{i}\right)$. If $G$ has this property, then it is also a basis for $I$, justifying the name. Another property of Gröbner bases is that for any given $f \in k\left[x_{1}, \ldots, x_{n}\right]$, 
there is a unique $r \in k\left[x_{1}, \ldots x_{n}\right]$ such that $L T\left(g_{i}\right)$ does not divide $L T(r)$ for any $i$, and

$$
f=q_{1} g_{1}+\ldots+q_{s} g_{s}+r
$$

although the $q_{i}$ 's may not be unique. This is the remainder of $f$ on division by $G, \bar{f}^{G}$. It is also sometimes called the normal form of $f$ with respect to $I$, and gives a unique way of writing $f$ under the projection mapping $\pi: k\left[x_{1}, \ldots, x_{n}\right] \rightarrow k\left[x_{1}, \ldots, x_{n}\right] / I$. This also means that a Gröbner basis for an ideal can be used to test membership in that ideal, by checking $\bar{f}^{G}=0$. There are many other uses for Gröbner bases as well, some of which are described in the next section.

Theorem 3.2.2 (The Hilbert Basis Theorem) Every ideal $I \subset k\left[x_{1}, \ldots, x_{n}\right]$ has a finite generating set.

This theorem is proved on pg 74 of [1]. It can be extended to show that every ideal has a finite Gröbner basis. Furthermore, there is an algorithm for constructing a Gröbner basis from a given generating set for $I$, called Buchberger's algorithm (see Chapter 2 of [1]), as described below.

Let $f$ and $g$ be non-zero polynomials in $k\left[x_{1}, \ldots, x_{n}\right]$; choose a monomial ordering on $\left\{x_{1}, \ldots, x_{n}\right\}$, and take $\alpha=\operatorname{deg}(f)$, and $\beta=\operatorname{deg}(g)$. Let $\gamma=$ $\left(\gamma_{1}, \ldots, \gamma_{n}\right) \in \mathbb{Z}_{\geq 0}^{n}$ be such that $\gamma_{i}=\max \left(\alpha_{i}, \beta_{i}\right)$ for $1 \leq i \leq n$. Then the least common multiple of $L M(f)$ and $L M(g)$ is $\mathbf{x}^{\gamma}$. 
Definition 3.2.3 The S-polynomial of $f$ and $g$ is:

$$
S(f, g)=\frac{\boldsymbol{x}^{\gamma}}{L T(f)} \cdot f-\frac{\boldsymbol{x}^{\gamma}}{L T(g)} \cdot g
$$

where $\boldsymbol{x}^{\gamma}$ is the least common multiple of $L M(f)$ and $L M(g)$.

Note that by construction, either this is zero, or the leading term of $S(f, g)$ is less than both $L T(f)$ and $L T(g)$. These S-pairs can then be used to construct the following algorithm for finding a Gröbner basis for $I=\left\langle f_{1}, \ldots, f_{r}\right\rangle$.

\subsubsection{Buchberger's algorithm}

1. Let $G_{0}:=\left\{f_{1}, \ldots, f_{r}\right\}$.

2. Given some $G_{k}$ : for all $f_{i}, f_{j} \in G_{k}$ with $i \neq j$, find $s:=S\left(f_{i}, f_{j}\right)$, and take the remainder on dividing by $G_{k}, s^{\prime}:=\bar{s}^{G_{k}}$. Let $\mathcal{S}$ be the set of all such nonzero $s^{\prime}$.

3. If $\mathcal{S}=\emptyset$, then finish. Otherwise, set $G_{k+1}:=G_{k} \cup \mathcal{S}$ and repeat from Step 2 .

4. Let $G_{\infty}:=\bigcup G_{k}$.

Theorem 3.2.4 This algorithm will always terminate after a finite number of steps. The result is $G_{\infty}$, a Gröbner basis for $I$.

As the algorithm terminates in a finite number of steps, the Gröbner basis produced is finite. However, it is not necessarily minimal, in the sense that 
it may contain unnecessary elements. Also, the result will depend on the monomial ordering used. 


\section{Chapter 4}

\section{Finite Group Actions}

\subsection{Invariant polynomials}

The results for invariants of finite groups are well established, with many of the founding results in the field being due to Hilbert, and the theory is more complete than in the general case. In particular, there are algorithms that always allow us to find a finite basis for the invariant subring.

For this chapter, we assume that the field $k$ is of characteristic 0 . Let $G \subset$ $G L(n, k)$, the general linear group of invertible linear transformations of $k^{n}$ for a field $k$, be a finite group with a matrix representation. Then the elements of $G$ act on polynomials in $k\left[x_{1}, \ldots, x_{n}\right]$ as shown:

$$
A: f\left(x_{1}, \ldots, x_{n}\right) \longmapsto f\left(A \cdot\left(x_{1}, \ldots, x_{n}\right)\right) \quad \text { for } A \in G
$$

The polynomial $f$ is invariant under $G$ if $f\left(x_{1}, \ldots, x_{n}\right)=f\left(A \cdot\left(x_{1}, \ldots, x_{n}\right)\right)$ for all $A \in G$, and the set of all such invariants is denoted $k\left[x_{1}, \ldots, x_{n}\right]^{G}$. This is a subring of $k\left[x_{1}, \ldots, x_{n}\right]$. 
Theorem 4.1.1 Hilbert's Finiteness Theorem Let $G$ be a finite group acting on $k\left[x_{1}, \ldots, x_{n}\right]$. Then $k\left[x_{1}, \ldots, x_{n}\right]^{G}$ is finitely generated.

\subsection{Syzygies}

Suppose $\left\{f_{1}, \ldots, f_{r}\right\}$ is a set of generators for an invariant subring $k\left[x_{1}, \ldots, x_{n}\right]^{G}$, and consider the ring $k\left[y_{1}, \ldots, y_{r}\right]$. Algebraic relations between $f_{1}, \ldots, f_{r}$ are called syzygies. The set of all such relations in $k\left[y_{1}, \ldots, y_{r}\right]$ gives rise to an ideal, called the syzygy ideal, or the ideal of relations:

$$
I_{F}=\left\{h \in k\left[y_{1}, \ldots, y_{r}\right]: h\left(f_{1}, \ldots, f_{r}\right)=0\right\}
$$

where $F=\left\{f_{1}, \ldots, f_{r}\right\}$.

Given $F$, this can be calculated directly using Gröbner basis methods:

Theorem 4.2.1 Let $F:=\left\{f_{1}, \ldots, f_{r}\right\}$, and $J_{F}:=\left\langle f_{1}-y_{1}, \ldots, f_{r}-y_{r}\right\rangle$. Then

1. $I_{F}=J_{F} \cap k\left[y_{1}, \ldots, y_{r}\right]$

2. Choose an elimination ordering in $k\left[x_{1}, \ldots, x_{n}, y_{1}, \ldots, y_{r}\right]$ where $x_{i}>y_{j}$ for $1 \leq i \leq n, 1 \leq j \leq r$. Let $G$ be a Gröbner basis for $J_{F}$. Then $G \cap k\left[y_{1}, \ldots, y_{r}\right]$ is a Gröbner basis for $I_{F}$ in the monomial ordering induced on $k\left[y_{1}, \ldots, y_{r}\right]$. 
This is proved on pg 340 of [1]. If $I_{F} \neq\{0\}$, then invariants cannot be expressed uniquely in terms of the generating set $\left\{f_{1}, \ldots, f_{r}\right\}$. Instead, they are isomorphic to a factor ring:

Lemma 4.2.2 If $k\left[x_{1}, \ldots, x_{n}\right]^{G}=k\left[f_{1}, \ldots, f_{r}\right]$, then

$$
k\left[y_{1}, \ldots, y_{r}\right] / I_{F} \cong k\left[x_{1}, \ldots, x_{n}\right]^{G}
$$

Given a known generating set $\left\{f_{1}, \ldots, f_{m}\right\}$ for an invariant ring $k\left[x_{1}, \ldots, x_{n}\right]^{G}$, and a general polynomial $f \in k\left[x_{1}, \ldots, x_{n}\right]$, there is a test to determine if $f$ is a $G$-invariant:

Lemma 4.2.3 Choose an elimination ordering with $x_{i}>y_{j}$ for all $1 \leq i \leq$ $n, 1 \leq j \leq m$. Let $B$ be a Gröbner basis for the ideal $\left\langle f_{1}-y_{1}, \ldots, f_{m}-y_{m}\right\rangle \subset$ $k\left[x_{1}, \ldots, x_{n}, y_{1}, \ldots, y_{m}\right]$, and let $g$ be the remainder of $f$ on division by $B$, $\bar{f}^{B}$. Then:

1. $f \in k\left[f_{1}, \ldots, f_{m}\right]$ iff $g \in k\left[y_{1}, \ldots, y_{m}\right]$

2. If $f \in k\left[f_{1}, \ldots, f_{m}\right]$, then $f=g\left(f_{1}, \ldots, f_{m}\right)$

The second part of this can be used to write invariants in terms of the known generating set. However, this representation need not be unique, depending on the syzygies between the generators. 


\subsection{Finding generators}

For any given $f \in k\left[x_{1}, \ldots, x_{n}\right]$, we can 'average out' the effects of $G$ :

Definition 4.3.1 Let $G$ be a finite matrix group. The Reynolds operator of $G$ is the mapping

$$
\begin{aligned}
& R_{G}: k\left[x_{1}, \ldots, x_{n}\right] \longrightarrow k\left[x_{1}, \ldots, x_{n}\right] \\
& \text { such that } \quad R_{G}(f)\left(x_{1}, \ldots, x_{n}\right)=\frac{1}{|G|} \sum_{A \in G} f\left(A \cdot\left(x_{1}, \ldots, x_{n}\right)\right)
\end{aligned}
$$

Lemma 4.3.2 $R_{G}$ has the properties that

1. $R_{G}$ is k-linear in $f$

2. For all $f \in k\left[x_{1}, \ldots, x_{n}\right]$, then $R_{G}(f) \in k\left[x_{1}, \ldots, x_{n}\right]^{G}$

3. $f \in k\left[x_{1}, \ldots, x_{n}\right]^{G}$ iff $R_{G}(f)=f$

So $R_{G}$ gives a way of finding invariants from arbitrary polynomials. Furthermore, $R_{G}(f)$ acting on monomials produces homogenous polynomials of the same total degree as the original, since the group action does not change the degree, and so $R_{G}(f)$ will have the same multi-degree as $f$. In fact, $R_{G}$ can be used to find a full generating set for the invariant subalgebra. (The following theorem is due to Emmy Noether): 
Theorem 4.3.3 For a finite matrix group $G$,

$$
k\left[x_{1}, \ldots, x_{n}\right]^{G}=k\left[R_{G}\left(\boldsymbol{x}^{\beta}\right):|\beta| \leq|G|\right]
$$

A proof of this is given in [1], pg 331. This proves the Hilbert Finiteness Theorem for finite groups (4.1.1). It means that we only need to consider $R_{G}$ as applied to a finite number of monomials in order to find a full generating set for $k\left[x_{1}, \ldots, x_{n}\right]^{G}$. However, the number of calculations needed may be very large, and many will be unnecessary, in that the result is zero, or an invariant already obtained. One way to reduce the number of calculations necessary is by knowing the number of invariants expected at each degree; that is, the dimension of $k\left[x_{1}, \ldots, x_{n}\right]_{d}^{G}$, the set of all homogenous invariants of degree $d$. This information is encoded in the Hilbert series for $G$ :

Definition 4.3.4 The Hilbert series of $k\left[x_{1}, \ldots, x_{n}\right]^{G}$ is defined as:

$$
\Phi_{G}(z)=\sum_{d=0}^{\infty} \operatorname{dim}\left(k\left[x_{1}, \ldots, x_{n}\right]_{d}^{G}\right) z^{d}
$$

For the rest of this section, we assume that $k$ is algebraically closed as well. The following theorem may be found in [13] as Theorem 2.2.1, where it is given for $k=\mathbb{C}$ :

Theorem 4.3.5 (Molien's Theorem) The Hilbert series of $k\left[x_{1}, \ldots, x_{n}\right]$ is given by

$$
\Phi_{G}(z)=\frac{1}{|G|} \sum_{A \in G} \frac{1}{\operatorname{det}\left(I_{n}-z A\right)}
$$


For an example of its application, taken from Chapter 2 of [13], let $G$ be the dihedral group $D_{6}=\left\{\iota, \delta, \ldots, \delta^{5}, \sigma \delta, \ldots, \sigma \delta^{5}\right\}$ acting on $\mathbb{C}[x, y, z]$, where:

$$
\iota=\left(\begin{array}{lll}
1 & 0 & 0 \\
0 & 1 & 0 \\
0 & 0 & 1
\end{array}\right), \quad \sigma=\left(\begin{array}{ccc}
1 & 0 & 0 \\
0 & -1 & 0 \\
0 & 0 & -1
\end{array}\right), \quad \delta=\left(\begin{array}{ccc}
\frac{1}{2} & \frac{-\sqrt{3}}{2} & 0 \\
\frac{\sqrt{3}}{2} & \frac{1}{2} & 0 \\
0 & 0 & 1
\end{array}\right)
$$

Using Molien's Theorem, the Hilbert series is

$$
\Phi_{G}(z)=1+2 z^{2}+3 z^{4}+5 z^{6}+\ldots
$$

So there are two algebraically independent invariants of degree 2 , say $f_{1}$ and $f_{2}$, which generate three invariants of degree $4: f_{1}^{2}, f_{1} f_{2}, f_{2}^{2}$, and four of degree 6: $f_{1}^{3}, f_{1}^{2} f_{2}, f_{1} f_{2}^{2}, f_{2}^{3}$. But since $\operatorname{dim}\left(\mathbb{C}[x, y, z]_{6}^{G}\right)=5$, there must be another invariant $f_{3}$ of degree 6 , algebraically independent of $f_{1}$ and $f_{2}$, which we can find by applying $R_{G}$ to the monomials of degree 6 . Hence this simplifies calculations with the Reynolds operator.

The Hilbert series can also be used to test if a set of invariants $\left\{f_{1}, \ldots, f_{r}\right\}$ generates $k\left[x_{1}, \ldots, x_{n}\right]^{G}$, by calculating $\Phi_{G}(z)$ and comparing it to the Hilbert series of $k\left[f_{1}, \ldots, f_{r}\right]$. The $f_{i}$ 's will be a generating set if and only if these two series are equal. If they are not equal then, taking the least degree $d$ at which they differ, there must be some invariants of degree $d$ that are not in $k\left[f_{1}, \ldots, f_{r}\right]$. The Reynolds operator can then be applied to monomials of degree $d$ to find more invariants of that degree, and these can be added to $\left\{f_{1}, \ldots, f_{r}\right\}$

The Hilbert series of $k\left[f_{1}, \ldots, f_{r}\right]$ is found using the following lemma: 
Lemma 4.3.6 Let $f_{1}, \ldots, f_{r}$ be algebraically independent homogenous elements of $k\left[x_{1}, \ldots, x_{n}\right]$ with degrees $d_{1}, \ldots, d_{r}$ respectively. Then the Hilbert series of $R:=k\left[f_{1}, \ldots, f_{r}\right]$ is

$$
H(R, z)=\sum_{n=0}^{\infty}\left(\operatorname{dim} R_{d}\right) z^{d}=\frac{1}{\left(1-z^{d_{1}}\right) \ldots\left(1-z^{d_{r}}\right)}
$$

This is proved as Lemma 2.2.3 in [13], for $k=\mathbb{C}$. For example of its application (also taken from Chapter 2 of Sturmfels):

Example 1 Let $G:=\left\{ \pm\left(\begin{array}{ll}1 & 0 \\ 0 & 1\end{array}\right), \pm\left(\begin{array}{cc}0 & -1 \\ 1 & 0\end{array}\right)\right\}$. We want to show that its invariant ring $\mathbb{C}\left[x_{1}, x_{2}\right]^{G}$ is generated by the invariants $I_{1}:=x_{1}^{2}+x_{2}^{2}, I_{2}:=$ $x_{1}^{2} x_{2}^{2}, I_{3}:=x_{1} x_{2}^{3}-x_{1}^{3} x_{2}$.

Using Molien's theorem, the Hilbert series of the invariant subalgebra $\mathbb{C}\left[x_{1}, x_{2}\right]^{G}$ is:

$$
\Phi^{G}(z)=\frac{1}{4}\left(\frac{1}{(1-z)^{2}}+\frac{1}{(1+z)^{2}}+\frac{2}{1+z^{2}}\right)=\frac{1+z^{4}}{\left(1-z^{2}\right)\left(1-z^{4}\right)}
$$

We can use the Gröbner basis method of Theorem 4.2.1 to determine that the syzygies of $\left\{I_{1}, I_{2}, I_{3}\right\}$ are generated by $I_{3}^{2}-I_{2} I_{1}^{2}+4 I_{2}^{2}$; i.e. $I_{3}^{2}=I_{1}^{2} I_{2}-4 I_{2}^{2}$. So every polynomial in $\mathbb{C}\left[I_{1}, I_{2}, I_{3}\right]$ can be written in the form $q\left(I_{1}, I_{2}\right)+$ $I_{3} r\left(I_{1}, I_{2}\right)$, and $\mathbb{C}\left[I_{1}, I_{2}, I_{3}\right]$ can be written as $\mathbb{C}\left[I_{1}, I_{2}\right] \oplus I_{3} \mathbb{C}\left[I_{1}, I_{2}\right]$.

Since $I_{1}$ and $I_{2}$ are algebraically independent homogenous polynomials, from the syzygy calculation, Lemma 4.3 .6 gives the Hilbert series of $\mathbb{C}\left[I_{1}, I_{2}\right]$ as:

$$
\begin{aligned}
H(R, z) & =\frac{1}{\left(1-z^{d_{1}}\right)\left(1-z^{d_{2}}\right)} \\
& =\frac{1}{\left(1-z^{2}\right)\left(1-z^{4}\right)}
\end{aligned}
$$


As the degree of $I_{3}$ is 4 , each element of degree $d$ in $\mathbb{C}\left[I_{1}, I_{2}\right]$ corresponds to an element of degree $d+4$ in $I_{3} \mathbb{C}\left[I_{1}, I_{2}\right]$. Hence $\operatorname{dim}\left(\mathbb{C}\left[I_{1}, I_{2}\right]\right)_{d}=\operatorname{dim}\left(I_{3} \mathbb{C}\left[I_{1}, I_{2}\right]\right)_{d+4}$, and so the Hilbert series of $I_{3} \mathbb{C}\left[I_{1}, I_{2}\right]$ is:

$$
H(R, z)=\frac{z^{4}}{\left(1-z^{2}\right)\left(1-z^{4}\right)}
$$

So the Hilbert series of $\mathbb{C}\left[I_{1}, I_{2}, I_{3}\right]$ is:

$$
H(R, z)=\frac{1+z^{4}}{\left(1-z^{2}\right)\left(1-z^{4}\right)}
$$

which is exactly $\Phi_{G}(z)$, meaning that the two subspaces are equal, and the set $\left\{I_{1}, I_{2}, I_{3}\right\}$ generates the invariant subalgebra.

\subsection{Hironaka decompositions}

Again, we assume that $k$ is algebraically closed.

Definition 4.4.1 Let $R=\oplus_{d=0}^{\infty} R_{d}$ be a graded algebra over a field $k=R_{0}$, and let $F:=\left\{f_{1}, \ldots, f_{r}\right\}$ be a set of homogenous elements in $R$. Then $F$ is $a$ homogenous system of parameters if

1. $f_{1}, \ldots, f_{r}$ are algebraically independent.

2. $R$ is finitely generated as a module over $k\left[f_{1}, \ldots, f_{r}\right]$.

Lemma 4.4.2 An invariant ring always has a homogenous system of parameters. 
In fact, for a finite group $G, R$ is freely generated as a module over $k\left[f_{1}, \ldots, f_{r}\right]$. This is due to the fact that the invariant ring is Cohen-Macaulay (see Section 2.3 of [13] for a more complete discussion). This means that an invariant $\operatorname{ring} R:=k\left[x_{1}, \ldots, x_{n}\right]^{G}$ can be written as $R=\oplus_{i=1}^{t} g_{i} k\left[f_{1}, \ldots, f_{r}\right]$ for a set $\left\{g_{1}, \ldots, g_{t}\right\}$ of homogenous invariants, and a set $\left\{f_{1}, \ldots, f_{r}\right\}$ of algebraically independent homogenous invariants.

This is called the Hironaka decomposition of $R$. The $f_{i}$ 's are primary invariants, and the $g_{j}$ 's are secondary invariants; together, they form a generating set for $R$. The decomposition also means that for any secondary invariant $g_{j}$, there is always a syzygy of the form $g_{j}^{2}=h\left(f_{1}, \ldots, f_{r}\right)$, for some polynomial $h$.

This decomposition is not unique, and various decompositions may differ from each other in terms of the degrees of the primary and secondary invariants. However, once a set of primary invariants with degrees $d_{1}, \ldots, d_{r}$ has been found, this gives some information about the secondary invariants (see Proposition 2.3.6 of [13]):

Lemma 4.4.3 If the primary invariants of $R$ in a particular Hironaka decomposition have degrees $d_{1}, \ldots, d_{r}$, then:

1. The number of secondary invariants is $\frac{d_{1} \ldots d_{r}}{|G|}$. 
2. The degrees (including multiplicity) of the secondary invariants are the exponents of

$$
\left(\Phi_{G}(z)\right)\left(\prod_{i=1}^{r}\left(1-z^{d_{i}}\right)\right)=z^{e_{1}}+\ldots+z^{e_{s}}
$$

Once a Hironaka decomposition has been found for $R$, then the Hilbert series can be calculated immediately:

Lemma 4.4.4 For an invariant subalgebra with Hironaka decomposition as above, the Hilbert series is

$$
H(R, z)=\frac{\left(\sum_{j=1}^{s} z^{\operatorname{deg} g_{j}}\right)}{\prod_{i=1}^{r}\left(1-z^{\operatorname{deg} f_{i}}\right)}
$$

See pg 40 of [13] for a proof. In the case of Example 1, the primary invariants

are $I_{1}$ and $I_{2}$, and the secondary invariant is $I_{3}$, with the Hilbert series obtained matching that the above lemma.

\subsection{Reductive groups}

Although finite groups have a well-established invariant theory, it only extends to infinite groups in some cases. Groups for which it does are called reductive groups. For cases where the base field is of characteristic 0, this may be defined as:

Definition 4.5.1 A group $G$ is linearly reductive if every rational linear representation of $G$ is completely reducible. 
Examples of reductive groups include the general linear group, and any semisimple group such as $S O(3)$. However, $S E(3)$ is not reductive, as the subspace of infinitesimal translations in $\mathfrak{s e}(3)$ is invariant in the adjoint representation, but has no invariant complement.

For reductive groups, Hilbert's finiteness theorem (4.1.1) still applies. Furthermore, we can find a unique Reynolds operator for each group action (by integrating over $G$ with respect to Haar measure) with the following properties:

Lemma 4.5.2 Let $G$ be a linear algebraic group acting on $\left\{x_{1}, \ldots, x_{n}\right\}$. Then there exists a linear mapping $R_{G}: k\left[x_{1}, \ldots, x_{n}\right] \rightarrow k\left[x_{1}, \ldots, x_{n}\right]^{G}$ such that

1. $R_{G}(f)=(f)$ for all $f \in k\left[x_{1}, \ldots, x_{n}\right]^{G}$.

2. $R_{G}(A f)=R_{G}(f)$ for all $f \in k\left[x_{1}, \ldots, x_{n}\right]$ and all $A \in G$.

A mapping of this sort will have the same properties as in Lemma 4.3.2, and the averaging operator defined for finite groups is an example of one of these mappings.

Theorem 4.3.3 does not generalise, but there is an algorithm for finding the generators of the invariant ring of a reductive group, which may be found in [3]. Furthermore, we can define the Hilbert series of a reductive group as before, and there is a form of Molien's theorem that applies in some cases: 
Theorem 4.5.3 (Molien-Weyl Theorem)

1. Let $G$ be a compact Lie group, $k=\mathbb{R}$ or $\mathbb{C}$, and $d \mu$ be normalised Haar measure on $G$. Then for $|z|<1$,

$$
H\left(k\left[x_{1}, \ldots, x_{n}\right]^{G}, z\right)=\int_{G} \frac{d \mu}{\operatorname{det}(1-z g)}
$$

2. Let $G$ be semi-simple, and $T$ be a maximal torus of a maximal compact subgroup of $G$. Take $\alpha_{1}, \ldots, \alpha_{d}$ to be roots of $G$ and $W$ its Weyl group. Then, if $d \mu$ is normalised Haar measure on $T$ and $|z|<1$ :

$$
H\left(k\left[x_{1}, \ldots, x_{n}\right]^{G}, z\right)=\frac{1}{|W|} \int_{T} \frac{\left(1-\alpha_{1}(g)\right) \ldots\left(1-\alpha_{d}(g)\right)}{\operatorname{det}(1-z g)} d \mu
$$

For $S O(3)$, we can take $T$ to be the subgroup of rotations around the $z$ axis [4]. Applying the second part of Theorem 4.5.3 gives the Hilbert series of $S O(3)$ as:

$$
H\left(\mathbb{R}\left[x_{1}, x_{2}, x_{3}\right]^{S O(3)}\right)=\frac{1}{1-z^{2}}
$$

Finally, the invariant ring of a reductive group still has a Hironaka decomposition, and hence its Hilbert series can be written in the form of Lemma 4.4.4. 


\section{Chapter 5}

\section{SAGBI Bases}

\section{$5.1 \quad$ Introduction}

When $S O(3)$ or $S E(3)$ is acting on $\mathfrak{s e}(3)$, the resulting set of invariants is a subalgebra, rather than an ideal, so Gröbner bases cannot be used. Instead, we can use SAGBI bases (Subalgebra Analogues to Gröbner Bases for Ideals), first defined by Robbiano and Sweedler [10], which have similarly useful properties. For example, they can be used to test for membership in the subalgebra for which they form a basis, as with Gröbner bases and ideals.

Furthermore, as with Gröbner bases, a SAGBI basis for a subalgebra generates that subalgebra. However, SAGBI bases are not always finite, as a subalgebra of a polynomial ring, unlike an ideal, does not need to be finitely generated. It is also possible for finitely-generated subalgebras to have a finite SAGBI basis with respect to one term ordering, but not with respect to another, or to have no finite SAGBI basis for any term ordering at all. 
There is a SAGBI basis test, given in [10], analogous to the Gröbner basis test, which determines if a given set is a SAGBI basis. This then leads into a method for constructing a SAGBI basis from a given generating set, similar to the one in Buchbager theory, as in Section 1. For a finite generating set, and a subalgebra that has a finite SAGBI basis, the method will terminate after a finite number of steps. However, in general it need not do so.

If $R$ is a commutative ring, and $E: R \longrightarrow \mathbb{N}$ is any function, the support of $E$ is supp $E=\{r \in R: E(r) \neq 0\}$. An exponent function of $R$ is one that has finite support. In this case, let $R^{E}:=\prod_{t \in T} t^{E(t)}$, where $T$ is any finite set containing supp $E$. If $E$ is the constant function 0 , with the empty set as its support, then $R^{E}$ is defined as 1 .

For a subset $S$ of $R$ containing supp $E, S^{E}$ can be defined similarly. Let $R=k\left[x_{1}, \ldots, x_{n}\right]$. Then $S^{E}$ is called an $S$ power product, and the set of all such power products is denoted by $P P(S)$.

Choose some monomial ordering and find the leading monomial $L M(b)$ of $b \in k\left[x_{1}, \ldots, x_{n}\right]$. Then $L M(P P(S))=P P(L M(S))$.

Definition 5.1.1 Let $B$ be a subalgebra of $k\left[x_{1}, \ldots, x_{n}\right]$ and $S \subset B$. $S$ is a SAGBI basis for $B$ if the lead term of every element of $B$ lies in $P P(L M(S))=L M(P P(S)$.

This means that for every non-constant $b \in B, L M(b)$ is a finite product of terms of the form $L M(s)$, where $s \in S$. Equivalently, $L M(b)$ is the leading 
monomial of a finite product of elements of $S$. Note that a SAGBI basis for an ideal is a Gröbner basis as well.

The process of SAGBI reduction, or subduction, of an element $b \in k\left[x_{1}, \ldots, x_{n}\right]$ over a subset $S \subset k\left[x_{1}, \ldots, x_{n}\right]$ is as follows:

1. Initialise: Set $b_{0}=b$

2. If $b_{i} \in k$, stop

3. If $L M\left(b_{i}\right) \notin P P(L M(S))$, stop

4. Otherwise, there is an exponent function $E_{i}$ on $S$ with $\operatorname{LM}\left(b_{i}\right)=$ $L M\left(S^{E_{i}}\right)$, since $L M\left(b_{i}\right) \in L M(P P(S))$. Let $\gamma_{i}$ be such that the leading monomials of $b_{i}$ and $\gamma_{i} S^{E_{i}}$ are the same, and let $b_{i+1}=b_{i}-\gamma_{i} S^{E_{i}}$.

5. Then either $b_{i+1}=0$ or $\operatorname{LM}\left(b_{i+1}\right)<L M\left(b_{i}\right)$.

6. Repeat from Step 2.

The output of this algorithm is called the final subductum of $b$ over $S$. It will terminate after a finite number of steps, as the degree of $b_{i}$ is strictly decreasing.

Lemma 5.1.2 If $S \subset B$, where $B$ is a subalgebra of $k\left[x_{1}, \ldots, x_{n}\right]$, then $b \in B$ iff the final subductum $c$ of $b$ is also in $B$. 
Proof. By construction, $b=c+\sum_{i=0}^{M-1} \gamma_{i} S^{E_{i}}$, for some $M \in N$. As all $s \in S$ are in $B$, and $B$ is a subalgebra, $S^{E_{i}} \in B$ for all $i$. Hence $b \in B$ iff $c \in B$.

\subsection{Constructing a SAGBI basis}

Definition 5.2.1 Let $A$ be a subset of $k\left[x_{1}, \ldots, x_{n}\right]$, containing the support of the exponent functions $E$ and $F$. The pair $\{E, F\}$ is an $A$ tête-a-tête if $L M\left(A^{E}\right)=L M\left(A^{F}\right)$.

These are analogous to the $S$-polynomials in Gröbner basis theory.

Hence $L T\left(A^{E}\right)=\rho L T\left(A^{F}\right)$ for some nonzero $\rho \in k$. Let $T(E, F):=A^{E}-$ $\rho A^{F}$, so that $L M(T(E, F))<L M\left(A^{E}\right)$.

Let $A$ be a subset $\left\{f_{1}, \ldots, f_{m}\right\}$ of $k\left[x_{1}, \ldots, x_{n}\right]$. Then a homomorphism $\phi$ : $k\left[y_{1}, \ldots, y_{m}\right] \longrightarrow k\left[x_{1}, \ldots, x_{n}\right]$ can be constructed by mapping $y_{i} \mapsto L T\left(f_{i}\right)$ for each $i$. The tête-a-têtes of $A$ are represented by the elements of the kernel of $\phi$. More precisely, each element of the kernel, on making the substitution $y_{i} \mapsto f_{i}$, is $T(E, F)$ for some tête-a-tête $\{E, F\}$ of $A$.

Definition 5.2.2 A set $\mathcal{T}$ generates the $S$ tête-a-têtes if $\mathcal{T}$ is a set of $S$ tête-a-têtes, and for any $S$ tête-a-tête $(E, F)$ there are $S$ tête-a-têtes $\left\{L_{i}, R_{i}\right\}$ and $m_{i} \in \mathbb{N}$ such that: 


$$
\begin{aligned}
& \text { for each } i, \quad\left\{L_{i}, R_{i}\right\} \in \mathcal{T} \\
& \text { and } E=\sum_{i} m_{i} L_{i}, \quad F=\sum_{i} m_{i} R_{i}
\end{aligned}
$$

In the previous example, a generating set for the tête-a-têtes would be represented by a generating set for the kernel of $\phi$.

\subsubsection{SAGBI basis construction algorithm}

Let $G$ be a subset of $k\left[x_{1}, \ldots, x_{n}\right]$.

1. Initialise: Set $G_{0}=G$

2. Induction on $G_{j}$ :

(a) Let $T_{j} \subset k\left[x_{1}, \ldots, x_{n}\right]$ generate the $G_{j}$ tête-a-têtes

(b) For each $(L, R) \in T_{j}$, let $f(L, R)$ be any final subductum of $T(L, R)$ over $G_{j}$

(c) Let $F_{j}$ be the set of all $f(L, R) \notin k$

(d) Set $G_{j+1}=G_{j} \cup F_{j}$

3. Set $G_{\infty}=\bigcup G_{j}$ 
Theorem 5.2.3 Let $G$ be as above, and $B$ be the subalgebra of $k\left[x_{1}, \ldots, x_{n}\right]$ that it generates. Then:

\section{1. $G_{\infty}$ is a $S A G B I$ basis for $B$}

2. $G_{j}$ is a $S A G B I$ basis for $B$ iff $G_{j}=G_{j+1}$

The proof is given as Theorem 3.5 in [10]. 


\section{Chapter 6}

\section{$S O(n)$ invariants}

In order to understand the invariants of $S E(3)$, it is helpful to know those of the subaction corresponding to $S O(3)$, as described in Section 1. This subaction is isomorphic to an action of $S O(3)$ on $\mathbb{R}^{3} \times \mathbb{R}^{3}$, as the adjoint action of $S O(3)$ is isomorphic to its standard action on $\mathbb{R}^{3}$, although this is only true in the three-dimensional case. The result for the invariants of the standard action of $S O(3)$ is well-established, and the derivation here due to Chapter 2 of Weyl [15].

Let $O(n)$ be the group of all orthogonal $n \times n$ matrices, which can be split into two connected components: $O^{-}(n)$, containing all elements of $O(n)$ with determinant -1 , and $S O(n)$, which could be written in an equivalent manner as $O^{+}(n)$.

Hence we can define even and odd invariants for the action of $O(n)$ on multiple vectors in $\mathbb{R}^{n}$. The first sort are standard $O(n)$ invariants, but the second 
have the property:

$$
f\left(A\left(\mathbf{x}_{1}, \ldots, \mathbf{x}_{m}\right)\right)=\operatorname{det}(A) f\left(\mathbf{x}_{1}, \ldots, \mathbf{x}_{m}\right)
$$

Both of these are invariants for $S O(n)$, though only the even invariants are true invariants for $O(n)$.

\subsection{The action on two vectors}

Lemma 6.1.1 The invariants of $O(n)$ acting on two vectors $\boldsymbol{x}$ and $\boldsymbol{y}$ are generated by the scalar products $\boldsymbol{x} \cdot \boldsymbol{x}, \boldsymbol{x} \cdot \boldsymbol{y}$, and $\boldsymbol{y} \cdot \boldsymbol{y}$.

Proof. Let $f(\mathbf{x}, \mathbf{y})$ be an invariant of $O(n)$. Choose any two fixed $\mathbf{x}$ and $\mathbf{y}$, and construct a Cartesian coordinate system $\left\{\mathbf{e}_{1}, \ldots, \mathbf{e}_{n}\right\}$ in $\mathbb{R}^{n}$ such that:

$$
\begin{aligned}
& \mathbf{x}=\alpha \mathbf{e}_{1} \\
& \mathbf{y}=\beta \mathbf{e}_{1}+\gamma \mathbf{e}_{2}
\end{aligned}
$$

The transformation between the original coordinate system and the new one is an orthogonal one, and so $f$ should be invariant under it:

$$
f(\mathbf{x}, \mathbf{y})=f\left(\mathbf{x}^{\prime}, \mathbf{y}^{\prime}\right)
$$

where

$$
\begin{aligned}
& \mathbf{x}^{\prime}=(\alpha, 0, \ldots, 0) \\
& \mathbf{y}^{\prime}=(\beta, \gamma, 0, \ldots, 0)
\end{aligned}
$$


So $f \in k[\alpha, \beta, \gamma]$. By construction, $\alpha^{2}=\mathbf{x}^{\prime} \cdot \mathbf{x}^{\prime}=\mathbf{x} \cdot \mathbf{x}$ (as the scalar product is invariant under $O(n))$. Likewise, $\alpha \beta=\mathbf{x}^{\prime} \cdot \mathbf{y}^{\prime}=\mathbf{x} \cdot \mathbf{y}$, and $\beta^{2}+\gamma^{2}=\mathbf{y}^{\prime} \cdot \mathbf{y}^{\prime}=$ $\mathbf{y} \cdot \mathbf{y}$. This means that:

$$
\begin{aligned}
& \alpha=\sqrt{\mathbf{x} \cdot \mathbf{x}} \\
& \beta=\frac{\mathbf{x} \cdot \mathbf{y}}{\sqrt{\mathbf{x} \cdot \mathbf{x}}} \\
& \gamma=\sqrt{\frac{(\mathbf{x} \cdot \mathbf{x})(\mathbf{y} \cdot \mathbf{y})}{(\mathbf{x} \cdot \mathbf{y})^{2}}}
\end{aligned}
$$

Now two further orthogonal transformations can be applied by changing the direction of either $\mathbf{e}_{1}$ or $\mathbf{e}_{2}$. The first corresponds to $\alpha \mapsto-\alpha$ and $\beta \mapsto$ $-\beta$, and the second to $\gamma \mapsto-\gamma$. Since $f$ is invariant under both these transformations, this means that if it is written as a sum of monomials of the form $M:=\alpha^{a} \beta^{b} \gamma^{c}$, then $c$ must be even for each monomial, as must the sum of $a$ and $b$.

If $a$ and $b$ are both even, then

$$
\begin{aligned}
M & =\left(\alpha^{2}\right)^{m}(\alpha \beta)^{2 n}\left(\beta^{2}\right)^{p}\left(\gamma^{2}\right)^{r} \\
& \in k\left[\mathbf{x} \cdot \mathbf{x}, \mathbf{x} \cdot \mathbf{y}, \mathbf{y} \cdot \mathbf{y}, \frac{(\mathbf{x} \cdot \mathbf{y})^{2}}{\mathbf{x} \cdot \mathbf{x}}\right]
\end{aligned}
$$

On the other hand, if $a$ and $b$ are both odd, then

$$
\begin{aligned}
M & =\left(\alpha^{2}\right)^{m}(\alpha \beta)^{2 n+1}\left(\beta^{2}\right)^{p}\left(\gamma^{2}\right)^{r} \\
& \in k\left[\mathbf{x} \cdot \mathbf{x}, \mathbf{x} \cdot \mathbf{y}, \mathbf{y} \cdot \mathbf{y}, \frac{(\mathbf{x} \cdot \mathbf{y})^{2}}{\mathbf{x} \cdot \mathbf{x}}\right]
\end{aligned}
$$

Hence $f:=F\left(\mathbf{x} \cdot \mathbf{x}, \mathbf{x} \cdot \mathbf{y}, \mathbf{y} \cdot \mathbf{y}, \frac{\mathbf{x} \cdot \mathbf{y}}{\mathbf{x} \cdot \mathbf{x}}\right)$, for some $F$. Similarly, by taking $\mathbf{y}:=\alpha \mathbf{e}_{1}$ and $\mathbf{x}:=\beta \mathbf{e}_{1}+\gamma \mathbf{e}_{2}$, it is possible to write $f$ in the form $f:=G\left(\mathbf{x} \cdot \mathbf{x}, \mathbf{x} \cdot \mathbf{y}, \frac{\mathbf{x} \cdot \mathbf{y}}{\mathbf{y} \cdot \mathbf{y}}\right)$. 
These can be rewritten as:

$$
\begin{aligned}
& f=\frac{F(\mathbf{x} \cdot \mathbf{x}, \mathbf{x} \cdot \mathbf{y}, \mathbf{y} \cdot \mathbf{y})}{(\mathbf{x} \cdot \mathbf{x})^{l}} \\
& f=\frac{G(\mathbf{x} \cdot \mathbf{x}, \mathbf{x} \cdot \mathbf{y}, \mathbf{y} \cdot \mathbf{y})}{(\mathbf{y} \cdot \mathbf{y})^{m}}
\end{aligned}
$$

for some $l, m \in \mathbb{N}_{\geq 0}$.

Now let $h(\xi, \nu, \lambda):=\lambda^{m} F(\xi, \nu, \lambda)-\xi^{l} G(\xi, \nu, \lambda)$. Then by construction, $h(\mathbf{x} \cdot$ $\mathbf{x}, \mathbf{x} \cdot \mathbf{y}, \mathbf{y} \cdot \mathbf{y})=0$

Since $\{\mathbf{x} \cdot \mathbf{x}, \mathbf{x} \cdot \mathbf{y}, \mathbf{y} \cdot \mathbf{y}\}$ are algebraically independent (which can be checked by a syzygy calculation as in Section 4$)$, this means that $h(\xi, \nu, \lambda)=0$.

Hence $\lambda^{m} F(\xi, \nu, \lambda)=\xi^{l} G(\xi, \nu, \lambda)$, so $F$ is divisible by $\xi^{l}$. Therefore:

$$
\begin{aligned}
f & =\frac{F(\mathbf{x} \cdot \mathbf{x}, \mathbf{x} \cdot \mathbf{y}, \mathbf{y} \cdot \mathbf{y})}{(\mathbf{x} \cdot \mathbf{x})^{l}} \\
& =F_{1}(\mathbf{x} \cdot \mathbf{x}, \mathbf{x} \cdot \mathbf{y}, \mathbf{y} \cdot \mathbf{y})
\end{aligned}
$$

for some $F_{1} \in k\left[x_{1}, x_{2}, x_{3}\right]$.

When $n>2$, there are no odd invariants for the action of $O(n)$ on two vectors, and so this is a basis for the $S O(n)$ invariants as well. However, in the case $n=2$, an extra invariant must be added: the determinant

$$
|\mathbf{x y}|=\left|\begin{array}{ll}
x_{1} & y_{1} \\
x_{2} & y_{2}
\end{array}\right|
$$

which is an odd invariant. This gives a complete basis for the set of $S O(n)$ invariants. The $n=2$ case then forms the base case for the action of $S O(n)$ on $m$ vectors, for a general $m$, which proceeds by induction. 


\subsection{The action on $m$ vectors}

If the vectors are $\left\{\mathbf{x}_{1}, \ldots, \mathbf{x}_{m}\right\} \subset \mathbb{R}^{n}$, with the $j^{\text {th }}$ coordinate of $\mathbf{x}_{i}$ being denoted by $x_{i j}$, then the result is:

Theorem 6.2.1 For $S O(n)$ acting on $m$ vectors in $\mathbb{R}^{n}$,

1. Every even invariant can be written as a polynomial in the scalar products $\boldsymbol{x}_{i} \cdot \boldsymbol{x}_{j}$

2. Every odd invariant is a sum of terms of the form

$$
\left[\boldsymbol{x}_{i_{1}} \ldots \boldsymbol{x}_{i_{n}}\right] g\left(\boldsymbol{x}_{1}, \ldots, \boldsymbol{x}_{m}\right)
$$

where $g$ is an even invariant, and

$$
\left[\boldsymbol{x}_{i_{1}} \ldots \boldsymbol{x}_{i_{n}}\right]:=\left|\boldsymbol{x}_{i_{1}} \ldots \boldsymbol{x}_{i_{n}}\right|=\left|\begin{array}{ccc}
x_{i_{1} 1} & \ldots & x_{i_{n} 1} \\
x_{i_{1} 2} & \ldots & x_{i_{n} 2} \\
\vdots & \ddots & \vdots \\
x_{i_{1} n} & \ldots & x_{i_{n} n}
\end{array}\right|
$$

is the bracket factor.

Let this theorem be denoted by $T_{n}^{m}$. The first stage of the proof is to show that $T_{n-1}^{n-1} \rightarrow T_{n}^{n-1}$, so that if the theorem is true for $(n-1)$ vectors in $\mathbb{R}^{n-1}$, it will be true for the same number of vectors in $\mathbb{R}^{n}$. The next two steps, $T_{n}^{n-1} \rightarrow T_{n}^{n} \rightarrow T_{n}^{m}$, for $m>n$, require the use of a construction known as Capelli's identity. 
The $m<n$ case is covered in $T_{n}^{n}$; if $f=f\left(\mathbf{x}_{1}, \ldots, \mathbf{x}_{m}\right)$ is an invariant, then it can be written as a function of $\mathbf{x}_{1}, \ldots, \mathbf{x}_{n}$ for $n>m$, and so $T_{n}^{n}$ applies. In this case, the only invariants are even ones.

\subsection{Capelli's identity}

This identity has two forms: the special and general case, which are both defined in terms of polarizations of a polynomial $f$ :

If $f$ is a polynomial in the coordinates of $\mathbf{x}_{1}, \ldots, \mathbf{x}_{m}$, we can write it as $f\left(\mathbf{x}_{1}, \ldots, \mathbf{x}_{m}\right)=f\left(x_{11}, \ldots, x_{m n}\right)$. Then by introducing a small perturbation in $\mathbf{x}_{1}$ :

$$
\begin{aligned}
f\left(\left(x_{11}, \ldots, x_{1 n}, x_{21}, \ldots, x_{m n}\right)+t\left(x_{11}^{\prime}, \ldots, x_{1 n}^{\prime}, 0, \ldots, 0\right)\right. \\
=f\left(x_{11}+t x_{11}^{\prime}, \ldots, x_{1 n}+t x_{1 n}^{\prime}, x_{21}, \ldots, x_{m n}\right) \\
=f\left(x_{11}, \ldots, x_{m n}\right)+t f_{1}\left(x_{11}, \ldots, x_{m n}, x_{11}^{\prime}, \ldots, x_{1 n}^{\prime}\right)+\ldots
\end{aligned}
$$

Then we can define:

Definition 6.3.1 The polarized polynomial of $f$ is

$$
D_{x_{1}^{\prime} x_{1}} f:=f_{1}\left(x_{11}, \ldots, x_{m n}, x_{11}^{\prime}, \ldots, x_{1 n}^{\prime}\right)=f\left(\boldsymbol{x}_{1}, \ldots, \boldsymbol{x}_{m}, \boldsymbol{x}_{1}^{\prime}\right)
$$

This can be written in terms of differentials:

$$
D_{x_{1}^{\prime} x_{1}} f=\frac{\partial f}{\partial x_{11}} x_{11}^{\prime}+\ldots+\frac{\partial f}{\partial x_{1 n}} x_{1 n}^{\prime}
$$


Similarly, for general $1 \leq i, j \leq m, D_{x_{i} x_{j}} f$ is defined as:

$$
D_{x_{i} x_{j}} f=\frac{\partial f}{\partial x_{j 1}} x_{i 1}+\ldots+\frac{\partial f}{\partial x_{j n}} x_{i n}
$$

If $f$ is an (even or odd) invariant of $O(n)$, then $D_{x_{i} x_{j}} f$ is likewise.

Theorem 6.3.2 (Capelli's Identity) If $\left\{\boldsymbol{x}_{1}, \ldots, \boldsymbol{x}_{m}\right\}$ are independent vectors in an $n$-dimensional vector space, and $m \geq n$, then

$$
\left|\begin{array}{cccc}
D_{m m}+(m-1) & \ldots & D_{m 2} & D_{m 1} \\
\vdots & \ddots & \vdots & \vdots \\
D_{2 m} & \ldots & D_{22}+1 & D_{21} \\
D_{1 m} & \ldots & D_{12} & D_{11}
\end{array}\right| f=\left\{\begin{array}{l}
0 \text { if } m>n \\
\left|\boldsymbol{x}_{1} \ldots \boldsymbol{x}_{n}\right| \Omega f \text { if } m=n
\end{array}\right.
$$

where

$$
\Omega:=\left|\begin{array}{ccc}
\frac{\partial}{\partial x_{11}} & \cdots & \frac{\partial}{\partial x_{1 n}} \\
\vdots & \ddots & \vdots \\
\frac{\partial}{\partial x_{m 1}} & \cdots & \frac{\partial}{\partial x_{m n}}
\end{array}\right|
$$

where $x_{i j}$ is the $j^{\text {th }}$ coordinate of $\boldsymbol{x}_{i}$, and $D_{i j}:=D_{x_{i} x_{j}}$.

The case $m=n$ is Capelli's special identity, while $m>n$ is the general identity. The proof for both cases can be found in Chapter 2, Section 4 of [15].

We now return to the proof of Theorem 6.2.1: 
Proof. For a set of vectors $\left\{\mathbf{x}_{1}, \ldots, \mathbf{x}_{n-1}\right\}$ in $\mathbb{R}^{n}$, let $f\left(\mathbf{x}_{1}, \ldots, \mathbf{x}_{n-1}\right)=$ $f\left(x_{11}, \ldots, x_{(n-1) n}\right)$ be a $S O(n)$ invariant depending upon them, and set:

$$
f_{0}\left(\begin{array}{ccc}
x_{11} & \ldots & x_{(n-1) 1} \\
\vdots & \ddots & \vdots \\
x_{1 n} & \ldots & x_{(n-1) n}
\end{array}\right)=f\left(\begin{array}{ccc}
x_{11} & \ldots & x_{(n-1) 1} \\
\vdots & \ddots & \vdots \\
x_{1(n-1)} & \ldots & x_{(n-1)(n-1)} \\
0 & \ldots & 0
\end{array}\right)
$$

For any given $\mathbf{x}_{1}, \ldots, \mathbf{x}_{n-1}$, it is possible to construct a vector $\mathbf{z} \neq 0$ perpendicular to all $\mathbf{x}_{i}$. Then a Cartesian coordinate system can be chosen such that the last basis vector $\mathbf{e}_{n}$ has direction $\mathbf{z}$.

In the new coordinate system, $\mathbf{x}_{i}$ can be written as $\overline{\mathbf{x}}_{i}=\bar{x}_{i 1} \mathbf{e}_{1}+\ldots+$ $\bar{x}_{i(n-1)} \mathbf{e}_{n-1}$, by construction, and so the last coordinate vanishes. This change of coordinate system can be represented by an orthogonal transformation $\tau \in O(n)$, and if det $\tau \neq 1$, we can reverse the direction of one of the basis vectors to get det $\tau=1$. Hence $\tau$ is an element of $S O(n)$ with $\overline{\mathbf{x}}_{i}=\tau\left(\mathbf{x}_{i}\right)$, and so $f$ is invariant under the change:

$$
f\left(\mathbf{x}_{1}, \ldots, \mathbf{x}_{n-1}\right)=f\left(\overline{\mathbf{x}}_{1}, \ldots, \overline{\mathbf{x}}_{n-1}\right)=f_{0}\left(\overline{\mathbf{x}}_{1}, \ldots, \overline{\mathbf{x}}_{n-1}\right)
$$

Since the new coordinates $\overline{\mathbf{x}}_{i}$ are zero in the last coordinate, the function $f$ is equal to $f_{0}$ in these coordinates.

If $f$ is an odd invariant, then $f \mapsto-f$ under the transformation

$$
\left(\begin{array}{cccc}
1 & 0 & \ldots & 0 \\
0 & \ddots & & \vdots \\
\vdots & & 1 & 0 \\
0 & \ldots & 0 & -1
\end{array}\right) \in O^{-}(n)
$$


Clearly $f_{0}$ is not changed when this transformation is applied to $f$, but since $f \mapsto-f$, this means that $f_{0}=-f_{0}$, and so $f_{0}=0$. Hence, from (6.24), $f=0$, and so $T_{n}^{n-1}$ is satisfied.

On the other hand, if $f$ is an even invariant, then so is $f_{0}$, and by $T_{n-1}^{n-1}$ it can be written in terms of the scalar products $\overline{\mathbf{x}}_{i} \cdot \overline{\mathbf{x}}_{j}$ :

$$
f_{0}\left(\overline{\mathbf{x}}_{1}, \ldots, \overline{\mathbf{x}}_{n-1}\right)=F\left(\begin{array}{ccc}
\overline{\mathbf{x}}_{1} \cdot \overline{\mathbf{x}}_{1} & \ldots & \overline{\mathbf{x}}_{1} \cdot \overline{\mathbf{x}}_{n-1} \\
\vdots & \ddots & \vdots \\
\overline{\mathbf{x}}_{n-1} \cdot \overline{\mathbf{x}}_{1} & \ldots & \overline{\mathbf{x}}_{n-1} \cdot \overline{\mathbf{x}}_{n-1}
\end{array}\right)
$$

Since the scalar products are $S O(n)$ invariants, $\mathbf{x}_{i} \cdot \mathbf{x}_{j}=\overline{\mathbf{x}}_{i} \cdot \overline{\mathbf{x}}_{j}$, and so by (6.24):

$$
f\left(\mathbf{x}_{1}, \ldots, \mathbf{x}_{n-1}\right)=F\left(\begin{array}{ccc}
\mathbf{x}_{1} \cdot \mathbf{x}_{1} & \ldots & \left.\mathbf{x}_{1} \cdot \mathbf{x}_{(} n-1\right) \\
\vdots & \ddots & \vdots \\
\mathbf{x}_{n-1} \cdot \mathbf{x}_{1} & \ldots & \mathbf{x}_{n-1} \cdot \mathbf{x}_{n-1}
\end{array}\right)
$$

and hence $T_{n}^{n-1}$ is satisfied.

Now we apply Capelli's identity to $f$. The process of polarization $D_{i j}$ turns an (even or odd) invariant $f$ into another (even or odd) invariant. So for $m=n$, Capelli's special identity shows that if $f$ is a relative invariant: i.e. $A \cdot f=\lambda f$ for some constant factor $\lambda$, then $\Omega f$ is also.

Let $f\left(\mathbf{x}_{1}, \ldots, \mathbf{x}_{m}\right)$ be a form with degree $r_{i}$ in each of its arguments $\mathbf{x}_{i}$. Its total degree is $r:=r_{1}+\ldots+r_{m}$, and we can use this to impose graded lexicographic ordering on the set of all forms in $\mathbf{x}_{1}, \ldots, \mathbf{x}_{m}$. With this ordering, $\Omega f$ is of lower order than $f$, due to the differentiation process. 
As for the left hand side of (6.22), the term in the polarization determinant due to the leading diagonal is:

$$
\left(D_{m m}+m-1\right) \ldots\left(D_{22}+1\right) D_{11}
$$

From the expression for $D_{i j}$ in (6.20), we get:

$$
D_{i i} f=\frac{\partial f}{\partial x_{i 1}} x_{i 1}+\ldots+\frac{\partial f}{\partial x_{i n}} x_{i n}=r_{i} f
$$

for a constant factor $r_{i}$, as $f$ is a polynomial in the $x_{i j}^{\prime} s$. Hence the action of $\left(D_{m m}+m-1\right) \ldots D_{11}$ on $f$ takes it to $\left(r_{m}+m-1\right) \ldots\left(r_{2}+1\right) r_{1} f=\rho f$ for some constant $\rho$, which is nonzero as long as $r_{1} \neq 0$; that is, when $f$ actually depends on $\mathbf{x}_{1}$.

For any other term in the expansion of the polarization determinant, any $\left(D_{i i}+i-1\right)$ terms it contains from the leading diagonal will only have the effect of multiplying $f$ by a constant factor, and so the action on $f$ of this term is:

$$
\rho^{*} D_{\beta_{r} \alpha_{r}} \ldots D_{\beta_{1} \alpha_{1}}
$$

where $\rho^{*}$ is a constant factor, $\alpha_{r}>\ldots>\alpha_{1}$, and $\left(\beta_{1}, \ldots, \beta_{r}\right)$ is a permutation of $\left(\alpha_{1}, \ldots, \alpha_{r}\right)$ with $\alpha_{i} \neq \beta_{i}$. By construction, $D_{\beta_{1} \alpha_{1}} f$ has degree one less in $\mathbf{x}_{\alpha_{1}}$ than $f$, but degree one greater in $\mathbf{x}_{\beta_{1}}$, so their total degrees are the same. The conditions on $\alpha_{1}, \ldots, \alpha_{r}$ and $\beta_{1}, \ldots, \beta_{r}$ mean that $\alpha_{1}<\beta_{1}$, and hence $D_{\beta_{1} \alpha_{1}} f$ is of lower order than $f$ in the ordering chosen. 
Let $f^{*}:=-\rho^{*} D_{\beta_{1} \alpha_{1}} f$ and $\mathcal{P}=D_{\beta_{r} \alpha_{r}} \ldots D_{\beta_{2} \alpha_{2}}$. Then Capelli's identity can be rewritten as:

$$
\rho f=\left\{\begin{array}{l}
\sum \mathcal{P} f^{*} \quad \text { for } m>n \\
\sum \mathcal{P} f^{*}+\left[\mathbf{x}_{1} \ldots \mathbf{x}_{n}\right] \Omega f \quad \text { for } m=n
\end{array}\right.
$$

where both $\Omega f$ and the $f^{*}$ s are of lower order than $f$, and are invariants if $f$ is an invariant.

Now suppose $m>n$ and choose a finite set of invariants $\varphi_{1}, \ldots, \varphi_{\ell}$, depending on $\mathbf{x}_{1}, \ldots, \mathbf{x}_{m}$, and assume that the subring they generate is closed under polarization: i.e., that every $D_{\beta \alpha} \varphi_{i}$ can be expressed in terms of the set of $\varphi_{j}$ 's. So it is closed under the $\mathcal{P}$ operators. Hence, if the invariants $f^{*}$ can be expressed in terms of the $\varphi_{i}$ 's then by Capelli's identity, $\rho f$ can be as well. So if $\rho \neq 0, f$ can be expressed in terms of the $\varphi_{i}$ 's also.

The process can then be repeated inductively by applying it to each $f^{*}$, and decomposing it using Capelli's identity. At each step, the rank of the invariants (with respect to the ordering chosen) decreases. The process will continue until the invariant being considered is independent of $\mathbf{x}_{1}$, as then $\rho=0$ in $(6.30)$.

The conclusion is that if every invariant that depends on the $(m-1)$ vectors $\mathbf{x}_{2}, \ldots, \mathbf{x}_{m}$ can be expressed in terms of the $\varphi_{i}$ 's that are independent of $\mathbf{x}_{1}$, then all invariants depending on $\mathbf{x}_{1}, \ldots, \mathbf{x}_{m}$ can be expressed in terms of the $\varphi_{i}$ 's. 
Furthermore, we can extend this to the $m=n$ case using Capelli's special identity. This requires another assumption: that the bracket factor $\left[\mathbf{x}_{1} \ldots \mathbf{x}_{n}\right]$ can be expressed in terms of the $\varphi_{i}$ 's. If this is the case, then we have established:

Theorem 6.3.3 Let $\varphi_{1}, \ldots, \varphi_{\ell}$ be a finite set of invariants of a linear group of degree $n$, under the conditions stated above. Then:

1. The set $\left\{\varphi_{1}, \ldots, \varphi_{\ell}\right\}$ will be a generating set for the invariants of the action on $m$ vectors $\boldsymbol{x}_{1}, \ldots, \boldsymbol{x}_{m}$ if the restricted set of $\varphi_{i}$ 's that depend on $\boldsymbol{x}_{1}, \ldots, \boldsymbol{x}_{n}$ is a generating set for the action on those vectors.

2. Futhermore, if the bracket factor $\left[\boldsymbol{x}_{1} \ldots \boldsymbol{x}_{n}\right]$ can be expressed in terms of the $\varphi_{i}$ 's, then the same is true for the $(n-1)$ vectors $\boldsymbol{x}_{1}, \ldots, \boldsymbol{x}_{n-1}$.

This means that the problem of finding generating invariants for the action on $m>n$ vectors can be reduced to finding them for the action on $n$ vectors. In the case of the $S O(n)$ action we are looking at, it means that $T_{n}^{n-1}$ implies $T_{n}^{n}$, by Capelli's special identity, and $T_{n}^{n}$ implies $T_{n}^{m}$ by the general identity. Here we are taking the set of $\phi_{i}$ 's to be the scalar products and the bracket factor. The only condition that needs to be checked is that $\left[\mathbf{x}_{1} \ldots \mathbf{x}_{n}\right] \Omega f$ can be written in terms of the scalar products $\mathbf{x}_{i} \cdot \mathbf{x}_{j}$. This is true because $\Omega f$ is an odd invariant of lower rank than $f$, and so by the induction hypothesis can be written as the product of $\left[\mathbf{x}_{1} \ldots \mathbf{x}_{n}\right]$ and a polynomial in the scalar 
products. Then

$$
\left[\mathbf{x}_{1} \ldots \mathbf{x}_{n}\right]^{2}=\left|\begin{array}{ccc}
\mathbf{x}_{1} \cdot \mathbf{x}_{1} & \ldots & \mathbf{x}_{1} \cdot \mathbf{x}_{n} \\
\vdots & \ddots & \vdots \\
\mathbf{x}_{n} \cdot \mathbf{x}_{1} & \ldots & \mathbf{x}_{n} \cdot \mathbf{x}_{n}
\end{array}\right|
$$

and so $\left[\mathbf{x}_{1} \ldots \mathbf{x}_{n}\right] \Omega f$ can be written entirely in terms of the scalar products.

Hence Theorem 6.2.1 is true for all $m$.

\subsection{A SAGBI basis}

The generating set from Theorem 6.2.1 can be extended to a SAGBI basis, as in Section 4, which has more useful properties. One possible way of doing this is given in Dalbec [2], taken from Richman [9]:

Let $X_{m \times n}$ be the matrix with $(i, j)^{t h}$ entry $x_{i j}$. Then $X_{m \times n} X_{m \times n}^{T}$ is the matrix of scalar products with $(i, j)^{t h}$ entry $\mathbf{x}_{i} \cdot \mathbf{x}_{j}$.

We can denote its minors by:

$$
f_{j_{1}, \ldots, j_{k}}^{i_{1}, \ldots, i_{k}}:=\left|\begin{array}{ccc}
\mathbf{x}_{i_{1}} \cdot \mathbf{x}_{j_{1}} & \ldots & \mathbf{x}_{i_{1}} \cdot \mathbf{x}_{j_{k}} \\
\vdots & \ddots & \vdots \\
\mathbf{x}_{i_{k}} \cdot \mathbf{x}_{j_{1}} & \ldots & \mathbf{x}_{i_{k}} \cdot \mathbf{x}_{j_{k}}
\end{array}\right|
$$

Theorem 6.4.1 The set of elements $f_{j_{1}, \ldots, j_{m}}^{i_{1}, \ldots, i_{m}}$ for $1 \leq k \leq n-1$, together with the determinants $\left|\boldsymbol{x}_{i_{1}} \ldots \boldsymbol{x}_{i_{n}}\right|$ for all $1 \leq i_{1}<\ldots<i_{n} \leq m$, form a SAGBI basis for the invariants of $S O(n)$, under lexicographic ordering $x_{11}>\ldots>x_{1 n}>x_{21}>\ldots>x_{m n}$. 
The proof can be found in [9]. Although only the scalar products and the determinants are needed to give a basis for the set of invariants, the $k \times k$ minors must be added to form a SAGBI basis, despite the fact that they can be generated from the scalar products. This is because cancellation occurs with the leading terms of the scalar products involved, and so the leading term of $f_{j_{1}, \ldots, j_{m}}^{i_{1}, \ldots, i_{m}}$ cannot be generated from the leading terms of the scalar products alone.

\subsection{The standard action of $S E(n)$}

The SAGBI basis above can be used to find a SAGBI basis for the standard affine action of $S E(3)$ on $m+1$ vectors. Although this is not the action that we are interested in, it demonstrates some of the useful properties of SAGBI bases, as well as a method for using a known set of $S O(n)$ invariants to find the $S E(n)$ ones. The derivation here is taken from [2], though there it is done for $O(n)$ and $E(n)$.

If $S O(n)$ is acting on the vectors $\mathbf{x}_{1}, \ldots, \mathbf{x}_{m}$ in $\mathbb{R}^{n}$, and $S E(n)$ is acting on $\mathbf{v}_{1}, \ldots, \mathbf{v}_{m+1}$, also in $\mathbb{R}^{n}$, then let $\phi$ be the algebra mapping:

$$
\phi: \mathbb{R}\left[x_{11}, \ldots, x_{m n}\right] \rightarrow \mathbb{R}\left[v_{11}, \ldots, v_{(m+1) n}\right]
$$

induced by the mapping $x_{i j} \mapsto v_{i j}-v_{(m+1) j}$.

The translational group gives a subaction of $S E(n)$, as described in Section 
1 , and $\phi\left(x_{i j}\right)$ is an invariant of that action, since for $\mathbf{r} \in \mathbb{R}^{n}$ :

$$
(0, \mathbf{r}) \cdot\left(v_{i j}-v_{(m+1) j}\right)=\left(v_{i j}+r_{j}\right)-\left(v_{(m+1) j}+r_{j}\right)=v_{i j}-v_{(m+1) j}
$$

It follows that $\phi(f)$ is a translational invariant for any $f \in \mathbb{R}\left[x_{11}, \ldots, x_{m n}\right]$. Furthermore, $\phi$ is injective, since if $\phi(f)\left(v_{11}, \ldots, v_{(m+1) n}\right)=0$, then $\phi(f)\left(v_{11}, \ldots, v_{m n}, 0, \ldots, 0\right)=0$ also. But this means $f\left(x_{11}, \ldots, x_{m n}\right)=0$, since they are the same under the substitution $x_{i j} \mapsto v_{i j}$.

Lemma 6.5.1 Let the $v_{i j}$ 's have a monomial ordering in which $v_{i j}>v_{(m+1) j}$ for $1 \leq i \leq m$ and $1 \leq j \leq n$. Then $B:=\left\{v_{i j}-v_{(m+1) j}: 1 \leq i \leq m, 1 \leq j \leq\right.$ $n\}$ is a SAGBI basis for the translational invariants.

See Lemma 2.1 of [2] for a proof. This then gives the result:

Lemma 6.5.2 $\phi$ is surjective as a mapping from the invariants of $S O(n)$ to the $S E(n)$ invariants.

Proof. Let $f \in \mathbb{R}\left[x_{11}, \ldots, x_{m n}\right]$ be an $S O(n)$ invariant. Then for any $(R, \mathbf{r}) \in$ $S E(n)$ :

$$
(R, \mathbf{r}) \cdot \phi(f)=(R, 0) \cdot \phi(f)
$$

as $\phi(f)$ is a translational invariant. Furthermore:

$$
(R, 0) \cdot \phi(f)=\phi(R \cdot f)=\phi(f)
$$


as $\phi$ is an algebra map, and $f$ is an $S O(n)$ invariant. So $\phi(f)$ is an $S E(3)$ invariant.

Now, let $h \in \mathbb{R}\left[v_{11}, \ldots, v_{(m+1) n}\right]$ be an $S E(n)$ invariant. By Lemma 6.5.2, it can be written in terms of some $\phi\left(x_{i j}\right) \mathrm{s}$, as it is a translational invariant. Furthermore, as $\phi$ is an algebra map, this means that $h=\phi(f)$ for some $f \in \mathbb{R}\left[x_{11}, \ldots, x_{m n}\right]$. Then:

$$
\phi(R \cdot f)=(R, 0) \cdot \phi(f)=(R, 0) h=h=\phi(f)
$$

as $h$ is a Euclidean invariant. Since $\phi$ is injective, this means that $R \cdot f=f$ $\forall R \in S O(3)$, and so $f$ is an $S O(n)$ invariant.

We can define some basic invariants for $S E(3)$, called the squared distances:

$$
D(i, j):=\left(v_{i 1}-v_{j 1}\right)^{2}+\ldots+\left(v_{i n}-v_{j n}\right)^{2}
$$

The scalar products $\mathbf{x}_{i} \cdot \mathbf{x}_{j}$ are mapped under $\phi$ to expressions in these $D(i, j)$ 's:

$$
\begin{gathered}
2 \phi\left(\mathbf{x}_{i} \cdot \mathbf{x}_{j}\right)=D(i, m+1)+D(j, m+1)-D(i, j) \\
\phi\left(\mathbf{x}_{i} \cdot \mathbf{x}_{i}+\mathbf{x}_{j} \cdot \mathbf{x}_{j} 2 \mathbf{x}_{i} \cdot \mathbf{x}_{j}\right)=D(i, j) \\
\phi\left(\mathbf{x}_{i} \cdot \mathbf{x}_{i}\right)=D(i, m+1)
\end{gathered}
$$




$$
\begin{aligned}
\text { e.g. } \quad 2 \phi\left(\mathbf{x}_{i} \cdot \mathbf{x}_{j}\right)= & 2\left(v_{i 1}-v_{(m+1) 1}\right)\left(v_{j 1}-v_{(m+1) 1}\right)+\ldots \\
& +2\left(v_{i n}-v_{(m+1) n}\right)\left(v_{j n}-v_{(m+1) n}\right) \\
= & 2\left(v_{(m+1) 1}^{2}+\ldots+v_{(m+1) n}^{2}\right)-2\left(v_{i 1} v_{(m+1) 1}-\right. \\
& \left.\left.+v_{j 1} v_{(m+1) 1}\right)+\ldots+v_{i n} v_{(m+1) n}+v_{j n} v_{(m+1) n}\right) \\
& +2\left(v_{i 1} v_{j 1}+\ldots+v_{i n} v_{j n}\right)
\end{aligned}
$$




$$
\begin{aligned}
= & \left(v_{i 1}^{2}-2 v_{i 1} v_{(m+1) 1}+v_{(m+1) 1}^{2}+\ldots\right. \\
& \left.+v_{i n}^{2}-2 v_{i n} v_{(m+1) n}+v_{(m+1) n}^{2}\right) \\
& +\left(v_{j 1}^{2}-2 v_{j 1} v_{(m+1) 1}+v_{(m+1) 1}^{2}+\ldots\right. \\
& \left.+v_{j n}^{2}-2 v_{j n} v_{(m+1) n}+v_{(m+1) n}^{2}\right) \\
& -v_{i 1}^{2}-v_{j 1}^{2}-\ldots-v_{i n}^{2}-v_{j n}^{2}+2 v_{i 1} v_{j 1}+\ldots \\
& +2 v_{i n} v_{j n} \\
= & \left(v_{i 1}-v_{(m+1) 1}\right)^{2}+\ldots+\left(v_{i n}-v_{(m+1) n}\right)^{2} \\
& +\left(v_{j 1}-v_{(m+1) 1}\right)^{2}+\ldots+\left(v_{j n}-v_{(m+1) n}\right)^{2} \\
& -\left(v_{i 1}-v_{j 1}\right)^{2}-\ldots-\left(v_{i n}-v_{j n}\right)^{2} \\
= & D(i, m+1)+D(j, m+1)-D(i, j)
\end{aligned}
$$

Then the functions $f_{j_{1}, \ldots, j_{k}}^{i_{1}, \ldots, i_{k}}$ are mapped under $\phi$ to:

$$
\phi\left(f_{j_{1}, \ldots, j_{k}}^{i_{1}, \ldots, i_{k}}\right)=2\left(\frac{-1}{2}\right)^{k}\left|\begin{array}{ccccc}
0 & 1 & 1 & \cdots & 1 \\
1 & D\left(i_{1}, j_{1}\right) & D\left(i_{1}, j_{2}\right) & \cdots & D\left(i_{1}, j_{k}\right) \\
1 & D\left(i_{2}, j_{1}\right) & D\left(i_{2}, j_{2}\right) & \cdots & D\left(i_{2}, j_{k}\right) \\
\vdots & \vdots & \vdots & \ddots & \vdots \\
1 & D\left(i_{k}, j_{1}\right) & D\left(i_{k}, j_{2}\right) & \cdots & D\left(i_{k}, j_{k}\right)
\end{array}\right|
$$

These are called the Cayley-Menger bideterminants.

Finally, the matrix $X_{m \times n}$ transforms under $\phi$ to $V_{m \times n}-V_{m+1}$, where $V_{m \times n}$ is the matrix with $(i, j)^{t h}$ entry $v_{i j}$, for $1 \leq i \leq m$ and $1 \leq j \leq n$, and $V_{m+1}$ is the matrix of the same size, with $(i, j)^{t h}$ entry $v_{(m+1) j}$. The $k \times k$ minors of 
$X_{m \times n}$, which are the determinants $\left|\mathbf{x}_{i_{1}}, \ldots, \mathbf{x}_{i_{k}}\right|$, are mapped to the minors of $V_{m \times n}-V_{m+1}$.

The last step of the argument is to check that the SAGBI basis for the $S O(n)$ invariants given in Theorem 6.4.1 will remain a SAGBI basis under the mapping $\phi$.

Lemma 6.5.3 Let the $v_{i j}$ 's have the pure lexographic order with $v_{11}>v_{12}>$ $\ldots>v(m+1) n$. Let $A$ be a subalgebra of $\mathbb{R}\left[x_{11}, \ldots, x_{m n}\right]$ and let $B$ be $a$ SAGBI basis of $A$. Then $\phi(B)$ is a SAGBI basis of $\phi(A)$.

Proof. Fix $f \in A$. Then $L T(f)=L T\left(\prod b_{i}\right)$ for some $b_{i} \in B$. Note that $L T\left(\phi\left(x_{i j}\right)\right)=v_{i j}$, by the monomial ordering chosen. Hence if $L T(f)<$ $L T(g)$, then

$$
L T(\phi(f))=L T\left(f\left(v_{11}, v_{12}, \ldots, v_{m n}\right)\right)<L T\left(g\left(v_{11}, v_{12}, \ldots, v_{m n}\right)\right)=L T(\phi(g))
$$

Thus the map $f \mapsto L T(\phi(f))$ is order-preserving and injective on monomials.

If $p$ is any monomial of $f$ except for $L T(f)$, then $L T(f)>p$, so $L T(\phi(L T(f)))>L T(\phi(p))$, since the mapping is order-preserving. Hence 
$L T(\phi(f))=L T(\phi(L T(f)))$, so

$$
\begin{aligned}
L T(\phi(f)) & =L T(\phi(L T(f))) \\
& =L T\left(\phi\left(L T\left(\prod b_{i}\right)\right)\right) \\
& =L T\left(\phi\left(\prod b_{i}\right)\right) \\
& =L T\left(\prod \phi\left(b_{i}\right)\right)
\end{aligned}
$$

Hence $\{\phi(b): b \in B\}$ forms a SAGBI basis for $\phi(A)$.

Theorem 6.5.4 With this ordering on the $v_{i j}$ 's, the $k \times k$ Cayley-Menger bideterminants for $3 \leq k \leq n+2$ and the $n \times n$ minors of $V_{m \times n}-V_{m+1}$ form a SAGBI basis for the algebra of $S E(n)$ invariants.

Proof. By applying the previous lemma to the SAGBI basis for the algebra of $S O(n)$ invariants.

\subsection{Syzygies of $S O(n)$}

It is also helpful to know the algebraic relations between these invariants, in order to better understand the structure of the invariant ring. Let $S O(n)$ have its standard action on $\mathbf{x}_{1}, \ldots, \mathbf{x}_{m} \in \mathbb{R}^{n}$. Then for the invariant scalar products $\mathbf{x}_{i} \cdot \mathbf{x}_{j}$, there is only one type of syzygy possible [15]. This is the equation:

$$
f_{j_{1}, \ldots, j_{k}}^{i_{1}, \ldots, i_{k}}=0, \quad \text { for } k \geq n+1
$$


The ideal of syzygies is generated by all those $f$ 's with $k=n+1$. Since the $O(n)$ invariants are generated solely by the scalar products, these are the only syzygies possible in this case. However, for the $S O(n)$ invariants there are two other types of syzygies involving the extra bracket factor invariant [5]:

$$
\begin{aligned}
g_{j_{1}, \ldots, j_{n}}^{i_{1}, \ldots, i_{n}} & :=\left[\mathbf{x}_{i_{1}} \ldots \mathbf{x}_{i_{n}}\right]\left[\mathbf{x}_{j_{1}} \ldots \mathbf{x}_{j_{n}}\right]-\left|\begin{array}{ccc}
\mathbf{x}_{i_{1}} \cdot \mathbf{x}_{j_{1}} & \ldots & \mathbf{x}_{i_{1}} \cdot \mathbf{x}_{j_{n}} \\
\vdots & \ddots & \vdots \\
\mathbf{x}_{i_{n}} \cdot \mathbf{x}_{j_{1}} & \ldots & \mathbf{x}_{i_{n}} \cdot \mathbf{x}_{j_{n}}
\end{array}\right|=0 \\
k_{j}^{i_{1}, \ldots, i_{n}} & :=\sum_{\lambda=1}^{n}(-1)^{\lambda-1}\left[\mathbf{x}_{i_{1}} \ldots \mathbf{x}_{i_{\lambda-1}} \mathbf{x}_{i_{\lambda+1}}, \ldots, \mathbf{x}_{i_{n}}\right] \mathbf{x}_{i_{\lambda}} \cdot \mathbf{x}_{j}=0
\end{aligned}
$$

where $1 \leq i_{1}<\ldots<i_{\lambda}<\ldots<i_{n} \leq m$ in $(6.45)$.

Theorem 6.6.1 The set of all relations of the form $f_{j_{1}, \ldots, j_{n+1}}^{i_{1}, \ldots, i_{n+1}}, g_{j_{1}, \ldots, j_{n}}^{i_{1}, \ldots, i_{n}}$, and $k_{j}^{i_{1}, \ldots, i_{n}}$, generates the syzygy ideal for the invariants of the standard action of $S O(n)$.

This is the second fundamental theorem for vector invariants, as applied to $S O(n)$ : namely, that the invariant subring can be expressed as a polynomial ring modulo a finitely generated ideal, as in Lemma 4.2.2. Domokos and Dresky [5] take this further by finding a Gröbner basis for the same ideal.

Let $H$ be the set of strictly increasing sequences with entries in $\{1, \ldots, m\}$, and give it the following partial ordering:

$$
a:=\left(a_{1}, \ldots, a_{s}\right) \leq\left(b_{1}, \ldots, b_{t}\right)=: b, \text { if } t \leq s \text { and } a_{1} \leq b_{1}, \ldots, a_{t} \leq b_{t}
$$


Let $a, b, c \in H$ be three sequences such that $a$ has length $n, b$ and $c$ have length $t \leq n$, and:

$$
a \leq\left(b_{1}, \ldots, b_{t-1}\right), \quad a_{t}>b_{t} \quad \text { and } \quad b \leq c
$$

Now let $S_{n+1}^{t}$ be the set of all permutations in $S_{n+1}$ that take both $\left\{b_{1}, \ldots, b_{t}\right\}$ and $\left\{a_{t}, \ldots, a_{n}\right\}$ to strictly increasing sequences; that is, monotone increasing permutations. Then we can define another type of syzygy:

$$
\begin{aligned}
h_{a, b, c}:=\sum_{\sigma \in S_{n+1}^{t}} \operatorname{sgn}(\sigma)\left[\mathbf{x}_{a_{1}} \ldots \mathbf{x}_{a_{t-1}} \mathbf{x}_{\sigma\left(a_{t}\right)} \ldots \mathbf{x}_{\sigma\left(a_{n}\right)}\right] \\
\cdot\left|\begin{array}{ccc}
\mathbf{x}_{\sigma\left(b_{1}\right)} \cdot \mathbf{x}_{c_{1}} & \ldots & \mathbf{x}_{\sigma\left(b_{1}\right)} \cdot \mathbf{x}_{c_{t}} \\
\vdots & \ddots & \vdots \\
\mathbf{x}_{\sigma\left(b_{t}\right)} \cdot \mathbf{x}_{c_{1}} & \ldots & \mathbf{x}_{\sigma\left(b_{t}\right)} \cdot \mathbf{x}_{c_{t}}
\end{array}\right|=0
\end{aligned}
$$

The syzygy $k_{j}^{i_{1}, \ldots, i_{n}}$ is a special case of this, with $t=1$.

Theorem 6.6.2 The syzygy ideal for the invariants of $S O(n)$ has a Gröbner basis given by the set of all possible $f_{j_{1}, \ldots, j_{n+1}}^{i_{1}, \ldots, i_{n+1}}, g_{j_{1}, \ldots, j_{n}}^{i_{1}, \ldots, i_{n}}$, and $h_{a, b, c}$, where $a, b, c \in$ $H$ satisfy (6.47).

The proof can be found in [5], where it is Theorem 2.1 .

For example, when $S O(3)$ is acting on four vectors in $\mathbb{R}^{3}, n=3$ and $m=4$. Hence there is one syzygy of the first type, being the $4 \times 4$ matrix $f_{1,2,3,4}^{1,2,3,4}$.

$$
\left|\begin{array}{llll}
\mathbf{x}_{1} \cdot \mathbf{x}_{1} & \mathbf{x}_{1} \cdot \mathbf{x}_{2} & \mathbf{x}_{1} \cdot \mathbf{x}_{3} & \mathbf{x}_{1} \cdot \mathbf{x}_{4} \\
\mathbf{x}_{2} \cdot \mathbf{x}_{1} & \mathbf{x}_{2} \cdot \mathbf{x}_{2} & \mathbf{x}_{2} \cdot \mathbf{x}_{3} & \mathbf{x}_{2} \cdot \mathbf{x}_{4} \\
\mathbf{x}_{3} \cdot \mathbf{x}_{1} & \mathbf{x}_{3} \cdot \mathbf{x}_{2} & \mathbf{x}_{3} \cdot \mathbf{x}_{3} & \mathbf{x}_{3} \cdot \mathbf{x}_{4} \\
\mathbf{x}_{4} \cdot \mathbf{x}_{1} & \mathbf{x}_{4} \cdot \mathbf{x}_{2} & \mathbf{x}_{4} \cdot \mathbf{x}_{3} & \mathbf{x}_{4} \cdot \mathbf{x}_{4}
\end{array}\right|=0
$$


For syzygies of type $g_{j_{1}, \ldots, j_{n}}^{i_{1}, \ldots, i_{n}}$, there are ten possibilities:

$$
\begin{array}{cccc}
g_{1,2,3}^{1,2,3} & g_{1,2,4}^{1,2,3} & g_{1,3,4}^{1,2,3} & g_{2,3,4}^{1,2,3} \\
g_{1,2,4}^{1,2,4} & g_{1,3,4}^{1,2,4} & g_{2,3,4}^{1,2,4} \\
g_{1,3,4}^{1,3,4} & g_{2,3,4}^{1,3,4} & \\
g_{2,3,4}^{2,3,4} & \\
\text { e.g. } \quad g_{1,2,4}^{1,2,3}=\left|\mathbf{x}_{1} \mathbf{x}_{2} \mathbf{x}_{3}\right|\left|\mathbf{x}_{1} \mathbf{x}_{2} \mathbf{x}_{4}\right|-\left|\begin{array}{lll}
\mathbf{x}_{1} \cdot \mathbf{x}_{1} & \mathbf{x}_{1} \cdot \mathbf{x}_{2} & \mathbf{x}_{1} \cdot \mathbf{x}_{4} \\
\mathbf{x}_{2} \cdot \mathbf{x}_{1} & \mathbf{x}_{2} \cdot \mathbf{x}_{2} & \mathbf{x}_{2} \cdot \mathbf{x}_{4} \\
\mathbf{x}_{3} \cdot \mathbf{x}_{1} & \mathbf{x}_{3} \cdot \mathbf{x}_{2} & \mathbf{x}_{3} \cdot \mathbf{x}_{4}
\end{array}\right|=0
\end{array}
$$

Finally, for the last type of syzygy, the number of possible $a, b, c$ that meet the necessary conditions of (6.47) is 14 :

$$
\begin{aligned}
& t=1 \quad a=(2,3,4), \quad b=(1) \\
& c=(1),(2),(3), \text { or }(4) \\
& t=2 \quad a=(1,3,4), \quad b=(1,2) \\
& c=(1,2),(1,3),(1,4),(2,3),(2,4), \text { or }(3,4) \\
& t=3 \quad a=(1,2,4), \quad b=(1,2,3) \\
& c=(1,2,3),(1,2,4),(1,3,4), \text { or }(2,3,4)
\end{aligned}
$$

For example, if $t=2, a=(1,3,4), b=(1,2)$, and $c=(1,2)$ : 
then the possible permutations in $S_{4}^{2}$ are:

$$
(1,2,3,4) \longmapsto(1,2,3,4), \quad(1,3,2,4), \quad(1,4,2,3)
$$

$$
(2,3,1,4), \quad(2,4,1,3), \quad(3,4,1,2)
$$

So the syzygy is:

$$
\begin{aligned}
& h_{a, b, c}=\sum_{\sigma \in S_{4}^{2}} \operatorname{sgn}(\sigma)\left|\mathbf{x}_{1} \mathbf{x}_{\sigma(3)} \mathbf{x}_{\sigma(4)}\right|\left|\begin{array}{ll}
\mathbf{x}_{\sigma(1)} \cdot \mathbf{x}_{1} & \mathbf{x}_{\sigma(1)} \cdot \mathbf{x}_{2} \\
\mathbf{x}_{\sigma(2)} \cdot \mathbf{x}_{1} & \mathbf{x}_{\sigma(2)} \cdot \mathbf{x}_{2}
\end{array}\right| \\
& =\left|\mathbf{x}_{1} \mathbf{x}_{3} \mathbf{x}_{4}\right|\left|\begin{array}{lll}
\mathbf{x}_{1} \cdot \mathbf{x}_{1} & \mathbf{x}_{1} \cdot \mathbf{x}_{2} \\
\mathbf{x}_{2} \cdot \mathbf{x}_{1} & \mathbf{x}_{2} \cdot \mathbf{x}_{2}
\end{array}\right|-\left|\mathbf{x}_{1} \mathbf{x}_{2} \mathbf{x}_{4}\right|\left|\begin{array}{lll}
\mathbf{x}_{1} \cdot \mathbf{x}_{1} & \mathbf{x}_{1} \cdot \mathbf{x}_{2} \\
\mathbf{x}_{3} \cdot \mathbf{x}_{1} & \mathbf{x}_{3} \cdot \mathbf{x}_{2}
\end{array}\right| \\
& +\left|\mathbf{x}_{1} \mathbf{x}_{2} \mathbf{x}_{3}\right|\left|\begin{array}{lll}
\mathbf{x}_{1} \cdot \mathbf{x}_{1} & \mathbf{x}_{1} \cdot \mathbf{x}_{2} \\
\mathbf{x}_{4} \cdot \mathbf{x}_{1} & \mathbf{x}_{4} \cdot \mathbf{x}_{2}
\end{array}\right|=0
\end{aligned}
$$

The rest of the terms are zero, as they feature a repeated vector in the bracket factor.

Now, by Theorem 6.6.2, these invariants form a Gröbner basis for the syzygy ideal. 


\section{Chapter 7}

\section{The SE(3) Invariants}

Returning to the adjoint action of $S E(3)$, there is a result in [12] that lets us use SAGBI bases to find generating sets for invariant subalgebras, even for non-reductive groups.

\subsection{A basis for invariants}

Let $G$ be an affine algebraic group acting algebraically on an affine variety $X$, with an action $\psi$.

$$
\psi: G \times X \rightarrow X \quad(g, x) \mapsto g x
$$

Suppose the elements of $G$ have coordinates $a_{1}, \ldots, a_{m}$, and the elements of $X$ have coordinates $x_{1}, \ldots, x_{n}$. The ideals of algebraic relations between the coordinates are $I(G)$ and $I(X)$ respectively. 
The function $\psi^{*}$ is defined as follows: If $f\left(x_{1}, \ldots, x_{n}\right)$ is a function on $X$, then $\psi^{*}(f)=f \circ \psi$. Hence $\psi^{*}\left(x_{i}\right)$ is the $i^{\text {th }}$ coordinate of the group action on $X, \psi(x)$.

The following theorem is from [14], although the form in which it is presented is taken from [12]:

Theorem 7.1.1 The ring of invariants of the action $\psi$ is

$$
\begin{aligned}
k\left[x_{1}, \ldots, x_{n}\right]^{G} & =k\left[\psi^{*}\left(x_{1}\right), \ldots, \psi^{*}\left(x_{n}\right)\right] \cap \frac{k\left[x_{1}, \ldots, x_{n}\right]}{I(X)} \\
& \subset \frac{k\left[a_{1}, \ldots, a_{m}, x_{1}, \ldots, x_{n}\right]}{I(G)+I(X)}
\end{aligned}
$$

Using SAGBI bases, this becomes:

Lemma 7.1.2 If $\left\{f_{1}, \ldots, f_{r}\right\}$ is a SAGBI basis for $k\left[\psi^{*}\left(x_{1}\right), \ldots, \psi^{*}\left(x_{n}\right)\right]$ with respect to an elimination order, where $a_{i}>x_{j}$ for all $1 \leq i \leq m, 1 \leq$ $j \leq n$, then $\left\{f_{1}, \ldots, f_{r}\right\} \cap k\left[x_{1}, \ldots, x_{n}\right] / I(X)$ is a SAGBI basis for $k[X]^{G}$, and hence a generating set.

Proof. Let $g \in k\left[\psi^{*}\left(x_{1}\right), \ldots, \psi^{*}\left(x_{n}\right)\right] \cap k\left[x_{1}, \ldots, x_{n}\right]$. Then $g \in k\left[f_{1}, \ldots, f_{n}\right]$, as $\left\{f_{1}, \ldots, f_{n}\right\}$ generates $k\left[\psi^{*}\left(x_{1}\right), \ldots, \psi^{*}\left(x_{n}\right)\right]$.

By the definition of a SAGBI basis:

$$
L T(g)=\prod_{i=1}^{r}\left(L T\left(f_{i}\right)\right)^{n_{i}} \quad \text { for some } n_{i} \in \mathbb{N} \cup\{0\}
$$


Hence, for all non-zero $n_{i}, L T\left(f_{i}\right) \in k\left[x_{1}, \ldots, x_{n}\right]$, as $L T(g) \in k\left[x_{1}, \ldots, x_{n}\right]$.

Since we are using an elimination order, this means that every term in $f_{i}$ is in $k\left[x_{1}, \ldots, x_{n}\right]$. So $g$ is generated by $f_{i}$ 's that are in $k\left[x_{1}, \ldots, x_{n}\right]$.

Furthermore, $L T(g) \in P P\left(L T\left(f_{i}\right)\right)$, so $\left\{f_{1}, \ldots, f_{n}\right\} \cap k\left[x_{1}, \ldots, x_{n}\right]$ is a SAGBI basis as required.

When this is combined with the algorithm from Chapter 5 for finding a SAGBI basis, it gives an algorithm for finding a generating set for the invariants of the action $\psi$.

As outlined in Chapter 5, the tête-a-têtes in the SAGBI basis algorithm are found by determining the kernel of the mapping

$$
\begin{aligned}
\phi: k\left[y_{1}, \ldots, y_{s}\right] & \rightarrow \frac{k\left[a_{1}, \ldots, a_{m}, x_{1}, \ldots, x_{n}\right]}{\langle L T(I(G))\rangle+\langle L T(I(X))\rangle} \\
y_{i} & \mapsto L T\left(f_{i}\right) \quad \text { for } i=1, \ldots, s
\end{aligned}
$$

This can be done by means of an algorithm, which is given in [10], but in simple cases, such as those considered here, it can be done by inspection. Another option, if $I(G)$ and $I(X)$ are trivial, is to treat it as the problem of finding all algebraic relations between the $L T\left(f_{i}\right)$ 's. This amounts to finding the syzygy ideal, which can be done using Gröbner basis methods as in Theorem 4.2.1. 


\subsection{Application to $S O(n)$}

Since the action of $S O(3)$ is a subaction of $S E(3)$, with a known SAGBI basis, it is useful to test the method of Lemma 7.1.2 on it. However, the SAGBI basis calculation fails to converge in this case. This can be demonstrated by looking at the calculations for $S O(2)$.

The action $\psi$ of $A \in S O(2)$ on $(\mathbf{u}, \mathbf{v}) \in \mathbb{R}^{2} \times \mathbb{R}^{2}$ is given by:

$$
\left(\begin{array}{ll}
a & b \\
c & d
\end{array}\right)(\mathbf{u}, \mathbf{v})=\left(\left(\begin{array}{l}
a u_{1}+b u_{2} \\
c u_{1}+d u_{2}
\end{array}\right),\left(\begin{array}{l}
a v_{1}+b v_{2} \\
c v_{1}+d v_{2}
\end{array}\right)\right)
$$

From Section 6, we know that the invariant subalgebra of this action has a SAGBI basis $\{\mathbf{u} \cdot \mathbf{u}, \mathbf{u} \cdot \mathbf{v}, \mathbf{v} \cdot \mathbf{v},|\mathbf{u} \mathbf{v}|\}$.

The action $\psi^{*}$ is given by:

$$
\begin{aligned}
& \psi^{*}\left(u_{1}\right)=a u_{1}+b u_{2} \\
& \psi^{*}\left(u_{2}\right)=c u_{1}+d u_{2} \\
& \psi^{*}\left(v_{1}\right)=a v_{1}+b v_{2} \\
& \psi^{*}\left(v_{2}\right)=c v_{1}+d v_{2}
\end{aligned}
$$

Furthermore, there is an ideal $I(S O(2))$ of algebraic relations between $\{a, b, c, d\}$, generated by $A^{T} A=\mathcal{I}_{n}$ and $\operatorname{det} A=1$. Using lexicographic ordering with $a>b>c>d$, and calculating in Maple, the set $\mathcal{G}:=$ $\left\{a-d, b+c, c^{2}+d^{2}-1\right\}$ is a Gröbner basis for this ideal, and hence the ideal $\langle L T(I(G))\rangle$ in (7.3) generated by the leading terms of $I(G)$ has as basis the set $\left\{a, b, c^{2}\right\}$. 
Now for the first step of the SAGBI basis calculation for $\mathbb{R}\left[\psi^{*}\left(u_{1}\right), \ldots, \psi^{*}\left(v_{2}\right)\right]$, each of the coordinate functions $\psi^{*}\left(u_{1}\right), \ldots, \psi^{*}\left(v_{2}\right) \in \frac{\mathbb{R}\left[a, \ldots, v_{2}\right]}{\langle I(S O(2))\rangle}$ must be reduced with respect to $\mathcal{G}$ (done using lexicographic ordering with $a>b>$ $c>d>u_{1}>u_{2}>v_{1}>v_{2}$ ), giving:

$$
\begin{aligned}
& f_{1}:=\psi^{*}\left(u_{1}\right)=-c u_{2}+d u_{1} \\
& f_{2}:=\psi^{*}\left(u_{2}\right)=c u_{1}+d u_{2} \\
& f_{3}:=\psi^{*}\left(v_{1}\right)=-c v_{2}+d v_{1} \\
& f_{4}:=\psi^{*}\left(v_{2}\right)=c v_{1}+d v_{2}
\end{aligned}
$$

So the homomorphism $\phi: \mathbb{R}\left[y_{1}, \ldots, y_{4}\right] \rightarrow \frac{\mathbb{R}\left[a, b, c, d, u_{1}, u_{2}, v_{1}, v_{2}\right]}{L T(I(S O(2)))}$ is determined by:

$$
\begin{aligned}
& y_{1} \longmapsto-c u_{2} \\
& y_{2} \longmapsto c u_{1} \\
& y_{3} \longmapsto-c v_{2} \\
& y_{4} \longmapsto c v_{1}
\end{aligned}
$$

As $\langle L T(I(S O 2))\rangle$ is generated by $\left\{a, b, c^{2}\right\}$, this means that the product of any two $y_{i}$ 's is in the kernel of $\phi$. So the kernel is generated by the set of all such possible products $y_{i} y_{j}$, each of which gives a polynomial $h_{i j}$ on making the substitution $y_{i} \mapsto L T\left(f_{i}\right)$. Furthermore, on doing the calculations in Maple, the leading term has a factor of $c d$, since any $c^{2}$ terms are reduced to $\left(1-d^{2}\right)$ by the algebraic relations in $\mathcal{G}$. As none of $f_{1}, f_{2}, f_{3}, f_{4}$ have a factor of $d$ in their leading terms, this cannot be reduced further by SAGBI 
subduction. So the new polynomials $h_{i j}$ are added to the set of $f_{i}$ 's for the next iteration.

So on the next iteration, $\phi$ maps $y_{i} \mapsto L T\left(f_{i}\right)$ and $z_{k \ell} \mapsto L T\left(h_{k \ell}\right) \in$ $c d \cdot \mathbb{R}\left[u_{1}, u_{2}, v_{1}, v_{2}\right]$, meaning that any product of two variables in $\left\{y_{1}, \ldots, z_{44}\right\}$ is in the kernel of $\phi$ as before. Then the same argument as above shows that all new polynomials generated have a factor of $c d^{2}$ in their leading terms, which cannot be subducted further.

Hence it is clear that the algorithm will not converge, as at every step it generates polynomials with increasing powers of $d$ in the leading term. So the method of Lemma 7.1.2 fails for $S O(2)$, and similarly for the general case of $S O(n)$.

This means that it cannot be applied directly to the invariants of $S E(3)$. For example, in the case of $S E(3)$ acting on a single screw $(\boldsymbol{\omega}, \mathbf{v}) \in \mathfrak{s e}(3)$, when the rotation matrix is $R$, there is a subaction $\boldsymbol{\omega} \mapsto R \boldsymbol{\omega}$ of $S O(3)$ on $\boldsymbol{\omega}$. So a SAGBI basis calculation for this action includes a calculation of a SAGBI basis for the $S O(3)$ subaction, which causes the algorithm to fail.

Instead, we split the action of $S E(3)$ up into its two component parts: the rotations and translations. The reason for doing this is that the set of $S E(3)$ invariants in $\mathfrak{s e}(3)$ is precisely the intersection of the sets of rotational and translational invariants. The rotational subaction has a known SAGBI basis, from Chapter6, and so we only need to find generators for the subring of translational invariants. 


\subsection{Single-screw case}

When the translation $\mathbf{t}=\left(t_{1}, t_{2}, t_{3}\right)^{T}$ is acting on a single screw, $(\boldsymbol{\omega}, \mathbf{v})$, the action $\psi^{*}$ is given by:

$$
\begin{aligned}
& f_{1}:=\psi^{*}\left(\omega_{1}\right)=\omega_{1} \\
& f_{2}:=\psi^{*}\left(\omega_{2}\right)=\omega_{2} \\
& f_{3}:=\psi^{*}\left(\omega_{3}\right)=\omega_{3} \\
& f_{4}:=\psi^{*}\left(v_{1}\right)=t_{2} \omega_{3}-t_{3} \omega_{2}+v_{1} \\
& f_{5}:=\psi^{*}\left(v_{2}\right)=-t_{1} \omega_{3}+t_{3} \omega_{1}+v_{2} \\
& f_{6}:=\psi^{*}\left(v_{3}\right)=t_{1} \omega_{2}-t_{2} \omega_{1}+v_{3}
\end{aligned}
$$

(using lexicographic ordering with $t_{1}>t_{2}>t_{3}>w_{1}>w_{2}>\ldots>v_{2}>v_{3}$ ).

This is the initial set of equations $G_{0}$ in the SAGBI basis algorithm. The set of tête-a-têtes of these equations is then the kernel of $\phi: y_{i} \mapsto L T\left(f_{i}\right)$, which in this case is the homomorphism:

$$
\begin{aligned}
& y_{1} \mapsto \omega_{1} \\
& y_{2} \mapsto \omega_{2} \\
& y_{3} \mapsto \omega_{3} \\
& y_{4} \mapsto t_{2} \omega_{3} \\
& y_{5} \mapsto-t_{1} \omega_{3} \\
& y_{6} \mapsto t_{1} \omega_{2}
\end{aligned}
$$

The kernel of this mapping is generated by the single element $\left\{y_{2} y_{5}+y_{3} y_{6}\right\}$. Substituting for the $y_{i}$ 's gives $-t_{1} \omega_{1} \omega_{3}+t_{3} \omega_{1} \omega_{2}+\omega_{2} v_{2}+\omega_{3} v_{3}$. This has the 
same leading term as $\psi^{*}\left(\omega_{1}\right) \psi^{*}\left(v_{1}\right)$, so subtracting this gives: $\omega_{1} v_{1}+\omega_{2} v_{2}+$ $\omega_{3} v_{3}=\boldsymbol{\omega} \cdot \mathbf{v}$. This cannot be subducted any further, so this iteration finishes.

The generating set of equations for $\mathbb{R}\left[\psi^{*}\left(\omega_{1}\right), \ldots, \psi^{*}\left(v_{3}\right)\right]$ is now:

$$
\begin{aligned}
& \psi^{*}\left(\omega_{1}\right)=\omega_{1} \\
& \psi^{*}\left(\omega_{2}\right)=\omega_{2} \\
& \psi^{*}\left(\omega_{3}\right)=\omega_{3} \\
& \psi^{*}\left(v_{1}\right)=t_{2} \omega_{3}-t_{3} \omega_{2}+v_{1} \\
& \psi^{*}\left(v_{2}\right)=-t_{1} \omega_{3}+t_{3} \omega_{1}+v_{2} \\
& \psi^{*}\left(v_{3}\right)=t_{1} \omega_{2}-t_{2} \omega_{1}+v_{3} \\
& f_{1}=\omega_{1} v_{1}+\omega_{2} v_{2}+\omega_{3} v_{3}
\end{aligned}
$$

and $\phi$ takes:

$$
\begin{aligned}
& y_{1} \mapsto \omega_{1} \\
& y_{2} \mapsto \omega_{2} \\
& y_{3} \mapsto \omega_{3} \\
& y_{4} \mapsto t_{2} \omega_{3} \\
& y_{5} \mapsto-t_{1} \omega_{3} \\
& y_{6} \mapsto t_{1} \omega_{2} \\
& z_{1} \mapsto \omega_{1} v_{1}
\end{aligned}
$$

On the next iteration, there are no new elements in the kernel of $\phi$, and so the algorithm finishes. Hence the set of translational invariants has a SAGBI basis given by $\left\{\psi^{*}\left(\omega_{1}\right), \ldots, \psi^{*}\left(v_{3}\right), \boldsymbol{\omega} \cdot \mathbf{v}\right\} \cap \mathbb{R}\left[\omega_{1}, \ldots, v_{3}\right]=\left\{\omega_{1}, \omega_{2}, \omega_{3}, \boldsymbol{\omega} \cdot \mathbf{v}\right\}$. 
Theorem 7.3.1 The invariant subring $\mathbb{R}\left[\omega_{1}, \ldots, v_{3}\right]$ for the adjoint action of $S E(3)$ on a single screw has a basis given by $\mathcal{S}:=\{\boldsymbol{\omega} \cdot \boldsymbol{\omega}, \boldsymbol{\omega} \cdot \boldsymbol{v}\}$.

Proof. Let $f$ be an $S E(3)$ invariant for the action on a single screw. Then it can be written as:

$$
f=\sum_{b \in \mathbb{N}} g_{b}\left(\omega_{1}, \omega_{2}, \omega_{3}\right)(\boldsymbol{\omega} \cdot \mathbf{v})^{b} \quad \text { where } g \in \mathbb{R}\left[x_{1}, x_{2}, x_{3}\right]
$$

as it is a translational invariant. Let $A=\left(\begin{array}{cc}R & 0 \\ 0 & R\end{array}\right) \in S O(3) . f$ must also be a rotational invariant:

$$
\begin{aligned}
A f & =\sum_{b \in \mathbb{N}} A g_{b}\left(\omega_{1}, \omega_{2}, \omega_{3}\right)(\boldsymbol{\omega} \cdot \mathbf{v})^{b} \\
& =f
\end{aligned}
$$

This means that $A g_{b}=g_{b}$ for all $b \in \mathbb{N}$. Hence $g_{b}$ is an invariant of $S O(3)$ acting on $\boldsymbol{\omega}$, for which the SAGBI basis is $\{\boldsymbol{\omega} \cdot \boldsymbol{\omega}\}$, from Section 5 .

So $g_{b} \in \mathbb{R}[\omega \cdot \omega]$. Hence the $S E(3)$ invariants are generated by $\{\boldsymbol{\omega} \cdot \boldsymbol{\omega}, \boldsymbol{\omega} \cdot \mathbf{v}\}$.

This result can also be found as Theorem 3.1 of [4], although the proof there is different.

\subsection{Double and triple screw cases}

Using the same method, generating sets can be found for the translational invariants in double and triple screw cases, and potentially higher. 
For a pair of screws, $\left\{\left(\boldsymbol{\omega}_{1}, \mathbf{v}_{1}\right),\left(\boldsymbol{\omega}_{2}, \mathbf{v}_{2}\right)\right\}$, let $\omega_{i j}$ and $v_{i j}$ be the $j^{\text {th }}$ coordinates of $\boldsymbol{\omega}_{i}$ and $\mathbf{v}_{i}$ respectively. Then a SAGBI basis for the translational invariants is given by:

$$
\begin{array}{lr}
\omega_{i j} & \text { for } 1 \leq i \leq 2, \quad 1 \leq j \leq 3 \\
\boldsymbol{\omega}_{i} \cdot \mathbf{v}_{i} & \text { for } 1 \leq i \leq 2 \\
\boldsymbol{\omega}_{1} \cdot \mathbf{v}_{2}+\boldsymbol{\omega}_{2} \cdot \mathbf{v}_{1} &
\end{array}
$$

Since $\boldsymbol{\omega}_{i} \cdot \mathbf{v}_{i}$ and $\boldsymbol{\omega}_{1} \cdot \mathbf{v}_{2}+\boldsymbol{\omega}_{2} \cdot \mathbf{v}_{1}$ are both rotational invariants as well, the same argument as in Theorem 7.3.1 gives:

Theorem 7.4.1 The invariant ring of $S E(3)$ acting on two screws is generated by $\left\{\boldsymbol{\omega}_{\boldsymbol{i}} \cdot \boldsymbol{\omega}_{\boldsymbol{j}}, \boldsymbol{\omega}_{\boldsymbol{i}} \cdot \boldsymbol{v}_{i}, \boldsymbol{\omega}_{1} \cdot \boldsymbol{v}_{2}+\boldsymbol{\omega}_{2} \cdot \boldsymbol{v}_{1}\right\}$ (for $1 \leq i, j \leq 2$ ).

In the case of the translational invariants of a set of three screws, $\left\{\left(\boldsymbol{\omega}_{1}, \mathbf{v}_{1}\right),\left(\boldsymbol{\omega}_{2}, \mathbf{v}_{2}\right),\left(\boldsymbol{\omega}_{3}, \mathbf{v}_{3}\right)\right\}$, the algorithm was only taken to the point where all new polynomials generated were in $k\left[\omega_{11}, \ldots, v_{33}\right]$. On inspection, every element in this set could be generated by the following polynomials:

$$
\begin{gathered}
\omega_{i j} \quad \text { for } 1 \leq i, j \leq 3 \\
\boldsymbol{\omega}_{i} \cdot \mathbf{v}_{i} \quad \text { for } 1 \leq i \leq 3 \\
\boldsymbol{\omega}_{i} \cdot \mathbf{v}_{j}+\boldsymbol{\omega}_{j} \cdot \mathbf{v}_{i} \text { for } 1 \leq i<j \leq 3 \\
z_{123}, \quad z_{231}, \quad z_{312} \\
z_{121}-z_{323}, \quad z_{232}-z_{131}, \quad z_{313}-z_{212} \\
\text { where } z_{i j k}=\left|\begin{array}{ccc}
\omega_{1 i} & \omega_{2 i} & \omega_{3 i} \\
\omega_{1 j} & \omega_{2 j} & \omega_{3 j} \\
v_{1 k} & v_{2 k} & v_{3 k}
\end{array}\right|
\end{gathered}
$$


These are all translationally invariant, and it is likely that they form a generating set for the translational invariants. As far as I know, the $z_{i j k}$ invariants have not been identified before. Note that in the case of $z_{i j i}-z_{k j k}$, neither $z_{i j i}$ or $z_{k j k}$ alone is a translational invariant. Furthermore, none of the final six invariants is a rotational invariant, and so the method used for the previous cases to determine $S E(3)$ invariants (assuming that we have a full listing of translational invariants) does not work. By comparison with the known set of $S O(3)$ invariants, however, the $S E(3)$ invariant $z_{123}+z_{231}+z_{312}$ can be found. This is a previously known invariant, equal to the sum $\left|\boldsymbol{\omega}_{1} \boldsymbol{\omega}_{2} \mathbf{v}_{3}\right|+\left|\boldsymbol{\omega}_{2} \boldsymbol{\omega}_{3} \mathbf{v}_{1}\right|+\left|\boldsymbol{\omega}_{3} \boldsymbol{\omega}_{1} \mathbf{v}_{2}\right|$ of $S O(3)$ invariants.

One possible alternative method for finding the $S E(3)$ invariants is given in [12], which states that:

Lemma 7.4.2 The intersection of two subalgebras $A=k\left[f_{1}, \ldots, f_{r}\right]$ and $B=k\left[g_{1}, \ldots, g_{s}\right]$ in a polynomial ring $k\left[x_{1}, \ldots, x_{n}\right]$ can be found by calculating a SAGBI basis for

$$
k\left[f_{1}, \ldots, f_{r}\right] \subset \frac{k\left[x_{1}, \ldots, x_{n}, y_{1}, \ldots, y_{s}\right]}{\left\langle y_{1}-g_{1}, \ldots, y_{s}-g_{s}\right\rangle}
$$

This could potentially be used to find the intersection of the subalgebras of rotational and translational invariants, although the algorithm may not terminate. There is also the problem of finding $\langle L T(I)\rangle$ for $I=\left\langle y_{1}-g_{1}, \ldots, y_{s}-g_{s}\right\rangle$, which requires a Gröbner basis computation that becomes very difficult as $s$ increases. 
Another possible method is to consider the rotations acting on the space of the translational invariants. Taking $R \in S O(3)$ to be a general rotation:

$$
R=\left(\begin{array}{lll}
a & b & c \\
d & e & f \\
g & h & k
\end{array}\right)
$$

The action of $R$ on the translational invariants that have been found is:

$$
\begin{aligned}
\omega_{i 1} \longmapsto & a \omega_{i 1}+b \omega_{i 2}+c \omega_{i 3} \\
\omega_{i 2} \longmapsto & d \omega_{i 1}+e \omega_{i 2}+f \omega_{i 3} \\
\omega_{i 3} \longmapsto & g \omega_{i 1}+h \omega_{i 2}+k \omega_{i 3} \\
& \begin{aligned}
z_{123} \longmapsto & g^{2} z_{231}+h^{2} z_{312}+k^{2} z_{123}+g k\left(z_{121}-z_{323}\right) \\
& +h k\left(z_{313}-z_{212}\right)+g h\left(z_{232}-z_{131}\right) \\
z_{231} \longmapsto & a^{2} z_{231}+b^{2} z_{312}+c^{2} z_{123}+a b\left(z_{232}-z_{131}\right) \\
& +b c\left(z_{313}-z_{212}\right)+a c\left(z_{121}-z_{323}\right) \\
z_{312} \longmapsto & d^{2} z_{231}+e^{2} z_{312}+f^{2} z_{123}+d e\left(z_{232}-z_{131}\right) \\
& +d f\left(z_{121}-z_{323}\right)+e f\left(z_{313}-z_{212}\right)
\end{aligned}
\end{aligned}
$$




$$
\begin{aligned}
z_{121}-z_{323} \longmapsto & 2 a g z_{231}+2 b h z_{312}+2 c k z_{123}+(a k+c g)\left(z_{121}-z_{323}\right) \\
& +(a h+b g)\left(z_{232}-z_{131}\right)+(b k+c h)\left(z_{313}-z_{212}\right) \\
z_{232}-z_{131} \longmapsto & 2 c f z_{123}+2 b e z_{312}+2 a d z_{231}+(a f+c d)\left(z_{121}-z_{323}\right) \\
& +(b f+c e)\left(z_{313}-z_{212}\right)+(a e+b d)\left(z_{232}-z_{131}\right) \\
z_{313}-z_{212} \longmapsto & 2 f k z_{123}+2 e h z_{312}+2 d g z_{231}+(d k+g f)\left(z_{121}-z_{323}\right) \\
& +(d h+e g)\left(z_{232}-z_{131}\right)+(e k+f h)\left(z_{313}-z_{212}\right)
\end{aligned}
$$

So the set of translational invariants is closed under this action, and hence we can apply Theorem 7.1.1 to it. The main problem with this approach is that it includes a subaction of $R$ on $\left(\boldsymbol{\omega}_{1}, \boldsymbol{\omega}_{2}, \boldsymbol{\omega}_{3}\right)$, which as described earlier, causes the SAGBI basis algorithm to fail. However, given that we already know the SAGBI basis for this action, it may be possible to use it to avoid this problem. 


\section{Bibliography}

[1] Cox, D., Little, J., O'Shea, D., Ideals, Varieties, and Algorithms, 2nd ed., Springer-Verlag, New York, 1997

[2] Dalbec, J., Straightening Euclidean Invariants, Ann. Math. Art. Intell, 13 (1995) 97-108

[3] Derksen, H., Kemper, G., Computational Invariant Theory, SpringerVerlag, New York, 2002

[4] Donelan, P., Selig, J., Introduction to Polynomial Invariants of Screw Systems, Victoria University of Wellington, School of Mathematics, Statistics and Computer Science Technical Report 06-02, (2006) 20pp

[5] Domokos, M., Drensky, V., Gröbner Bases for the Rings of Special Orthogonal and $2 \times 2$ matrix Invariants, J. Algebra, 243 (2001) 706-716

[6] Hall, B., Lie Groups, Lie Algebras, and Representations, Graduate Texts in Mathematics, Vol. 222, Springer, 2004

[7] Hochster, M., Roberts, J.L., Rings of invariants of reductive groups acting on regular rings are Cohen-Macaulay, Adv. Math.,13 (1974) 115-175 
[8] Hunt, K. H., Kinematic Geometry of Mechanisms, Claredon Press, Oxford, 1978

[9] Richman, D., The Fundamental Theorems of Vector Invariants, Adv. Math., 73 (1989) 43-78

[10] Robbiano L., Sweedler, M., Subalgebra Bases, in: Bruns, W., Simis, A., (eds.) Commutative Algebra, Lecture Notes in Mathematics 1430, Springer, 1988, pp. 61-87

[11] Selig, J., Geometric Fundamentals of Robotics, Springer, New York, 2005

[12] Stillman, M., Tsai, H., Using SAGBI Bases to Compute Invariants, $J$. Pure Appl. Alg., 139 (1999) 285-302

[13] Sturmfels, B., Algorithms in Invariant Theory, Springer-Verlag, New York, 1993

[14] Vasconcelos, W., Computational Methods in Commutative Algebra and Algebraic Geometry, Springer, Berlin, 1997.

[15] Weyl, H., The Classical Groups: Their Invariants and Representations, Princeton University Press, Princeton, 1946 